\title{
Summary Report for the SINBAD Search Tool Project
}

\section{June 2012}

Prepared by

Alice Cunha da Silva, Federal University of Rio de Janeiro (UFRJ)

Work performed as part of the author's two-month practicum assignment at ORNL under mentorship by L. C. Leal through the ORNL Nuclear Science and Engineering Directorate Educational Outreach Program. Financial support for the author provided by UFRJ.

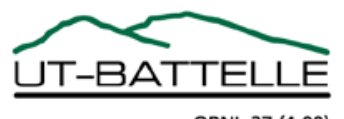

ORNL-27 (4-00) 


\section{DOCUMENT AVAILABILITY}

Reports produced after January 1, 1996, are generally available free via the U.S. Department of Energy (DOE) Information Bridge.

Web site http://www.osti.gov/bridge

Reports produced before January 1, 1996, may be purchased by members of the public from the following source.

National Technical Information Service

5285 Port Royal Road

Springfield, VA 22161

Telephone 703-605-6000 (1-800-553-6847)

TDD 703-487-4639

Fax 703-605-6900

E-mail info@ntis.gov

Web site http://www.ntis.gov/support/ordernowabout.htm

Reports are available to DOE employees, DOE contractors, Energy Technology Data Exchange (ETDE) representatives, and International Nuclear Information System (INIS) representatives from the following source.

Office of Scientific and Technical Information

P.O. Box 62

Oak Ridge, TN 37831

Telephone 865-576-8401

Fax 865-576-5728

E-mail reports@osti.gov

Web site http://www.osti.gov/contact.html

This report was prepared as an account of work sponsored by an agency of the United States Government. Neither the United States Government nor any agency thereof, nor any of their employees, makes any warranty, express or implied, or assumes any legal liability or responsibility for the accuracy, completeness, or usefulness of any information, apparatus, product, or process disclosed, or represents that its use would not infringe privately owned rights. Reference herein to any specific commercial product, process, or service by trade name, trademark, manufacturer, or otherwise, does not necessarily constitute or imply its endorsement, recommendation, or favoring by the United States Government or any agency thereof. The views and opinions of authors expressed herein do not necessarily state or reflect those of the United States Government or any agency thereof. 
ORNL/TM-2012/63

Reactor and Nuclear Systems Division

\title{
Summary Report for The SINBAD Search Tool Project
}

\author{
Alice Cunha da Silva, Federal University of Rio de Janeiro \\ Work performed as part of the author's two-month practicum assignment at ORNL under mentorship by \\ L. C. Leal through the ORNL Nuclear Science and Engineering Directorate Educational Outreach \\ Program. Financial support for the author provided by UFRJ.
}

Date Published: June 2012

\author{
Prepared by \\ OAK RIDGE NATIONAL LABORATORY \\ P.O. Box 2008 \\ Oak Ridge, Tennessee 37831-6285 \\ managed by \\ UT-BATTELLE, LLC \\ for the \\ U.S. DEPARTMENT OF ENERGY \\ under contract DE-AC05-00OR22725
}





\section{CONTENTS}

\section{Page}

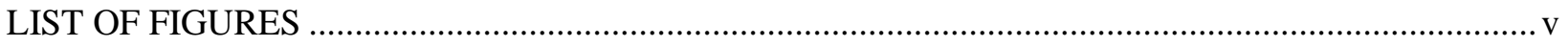

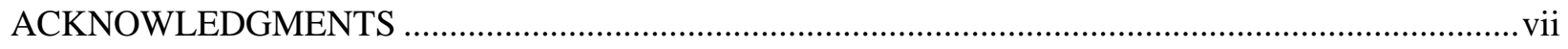

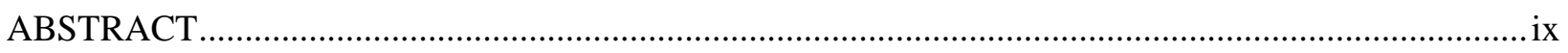

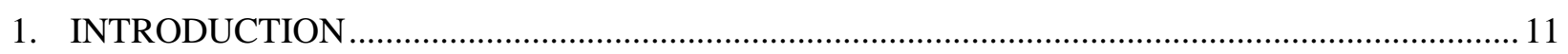

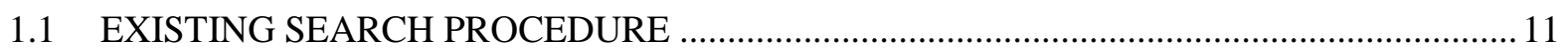

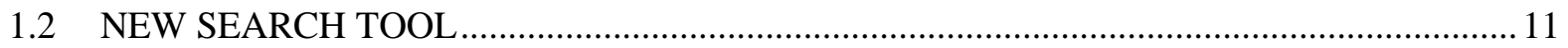

1.3 THE INTEGRATED DEVELOPMENT ENVIRONMENT (IDE) ....................................... 11

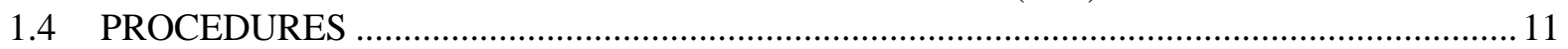

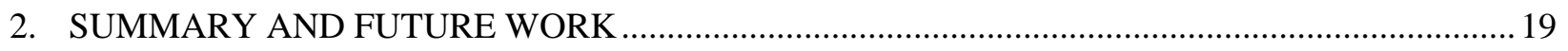

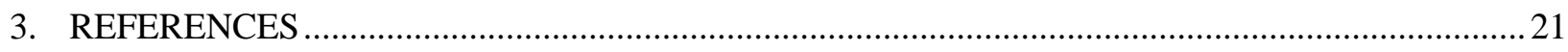

APPENDIX A : EXAMPLE OF THE PREVIOUS SEARCH METHOD ............................................A-1

APPENDIX B : LIST OF THE SINBAD SEARCH TOOL CODE, WRITTEN IN JAVA

LANGUAGE ON THE NetBeans IDE 7.1 PLATFORM......................................... B-1 



\section{LIST OF FIGURES}

Page

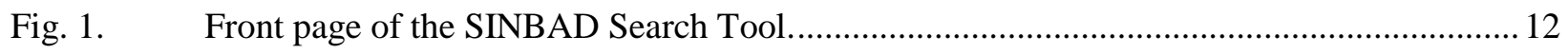

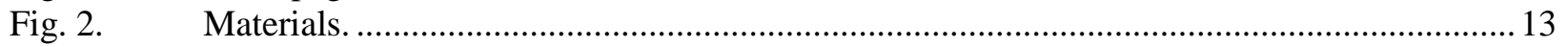

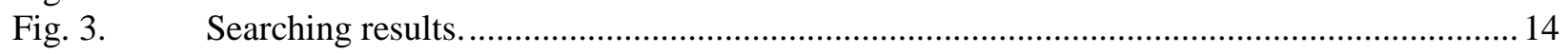

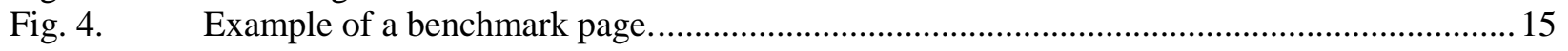

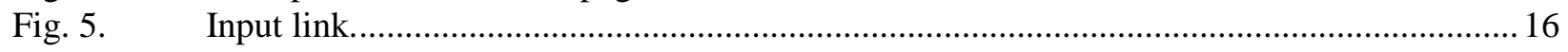

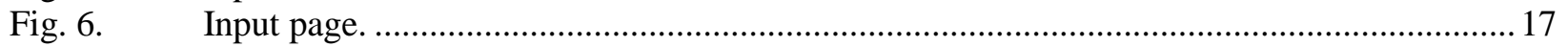

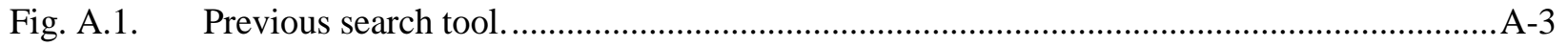

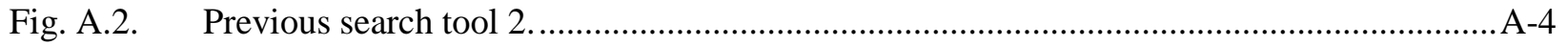

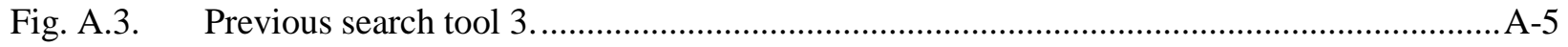

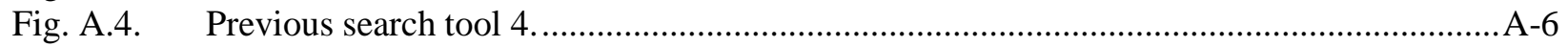

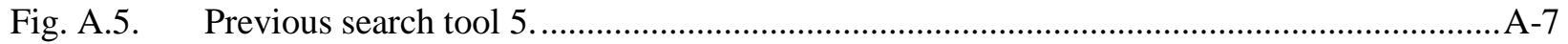

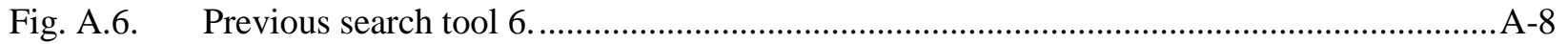

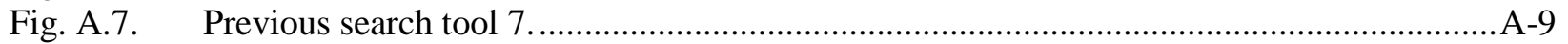

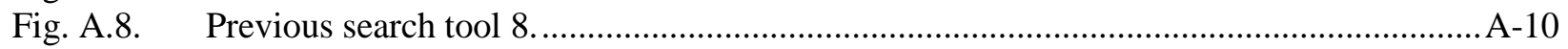

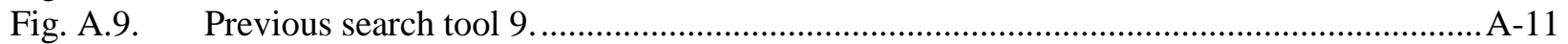

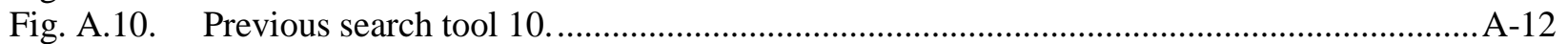

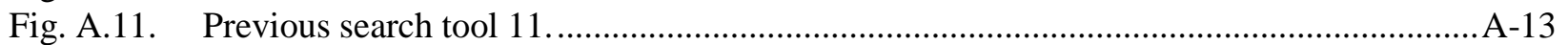

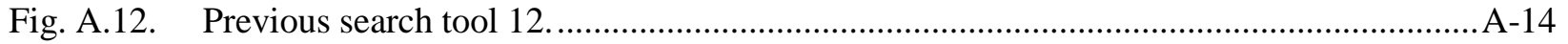





\section{ACKNOWLEDGMENTS}

This work was performed as part of an educational practicum assignment with the Nuclear Data and Criticality Safety Group of the Reactor and Nuclear Systems Division of the Oak Ridge National Laboratory (ORNL). The author is particularly indebted to Luiz Leal and Mike Dunn (ORNL) and Su Jian (Federal University of Rio de Janeiro) for their support. The author would also like to thank Robert Lefebvre and Dorothea Wiarda (ORNL) for their invaluable help. 



\begin{abstract}
The Shielding Integral Benchmark Archive Database (SINBAD) Search Tool has been developed to serve as an interface with the SINBAD database to facilitate a simple and quick means of searching for information related to experimental benchmark problems. The Search Tool is written in Java and provides a better and efficient way to retrieve information from the SINBAD database. Searches can be performed quickly and easily. With regard to improvements, users are no longer required to know the name of the benchmarks to search the database. Instead, a search can be performed by specifying the experimental facility, constituents of the experimental benchmark, etc. In summary, a new powerful database search tool has been developed for SINBAD.
\end{abstract}





\section{INTRODUCTION}

The Shielding Integral Benchmark Archive Database ${ }^{1,2}$ (SINBAD) includes benchmark experiments relevant to reactor shielding, fusion, and accelerator shielding applications. The database stores and makes available information related to high-quality benchmark experiments, which is useful for validating radiation transport codes and nuclear data. Several benchmark experiments have been incorporated into SINBAD. Every year new experiments are proposed for inclusion in the SINBAD database. The Radiation Safety Information Computational Center (RSICC) ${ }^{3}$ at Oak Ridge National Laboratory (ORNL) is responsible for collecting, evaluating, and distributing computer codes and databases pertinent to radiological study, including SINBAD.

The purpose of this work is to describe a tool, the SINBAD Search Tool that can be used for the simple and quick selection of documents and benchmarks resident in the SINBAD database.

\subsection{EXISTING SEARCH PROCEDURE}

The SINBAD system distributed by RSICC includes a very limited search tool that was developed in the HTML language. To find needed information with the existing tool, one must know the precise name of the benchmark (i.e., the user will have to look at each name of the benchmarks in the database). An example of the use of this tool is given in Appendix A.

\subsection{NEW SEARCH TOOL}

To improve the searching procedure of information in the SINBAD database, a new tool has been developed in the programming language Java. ${ }^{4}$ This language offers several features including the ability to execute procedures in various operational systems. After examining the structure of the SINBAD database, it was decided that the Java language was suitable for the project.

\subsection{THE INTEGRATED DEVELOPMENT ENVIRONMENT (IDE)}

For this project the NetBeans IDE $7.1^{5}$ was chosen because it is a free open-source Integrated Development Environment for software development, with all the tools needed to create professional desktop, enterprise, web, and mobile applications with the Java platform.

\subsection{PROCEDURES}

The search tool developed in this work consists of a graphical user interface (GUI) that permits the users to choose from a frame of options the information that better qualifies the benchmark search. As a result, the SINBAD Search Tool returns a list of benchmark names that matches the user's request. Examples of the search procedure using the search tool are displayed in Figs.1-6. The first page of the search tool is shown in Fig. 1. As can be seen, there are four boxes labeled Type, Material, Laboratory, and Geometry. Descriptions of each parameter are as follows:

Type: The user chooses the type of benchmark application, namely, Reactor Shielding, Fusion Neutronic Shielding, or Accelerator Shielding, of interest. If “All” is selected, all three options are retrieved simultaneously.

Material: The user provides the name of the material of interest. More than one material may be specified as shown in Fig. 2. This is the only field in which the user can choose more than one option. The method of doing so is operating-system dependent. On a Windows machine, the user must press the "Ctrl" key while pressing the second, third, and other choices. Selecting "Any" retrieves all options simultaneously. 
Laboratory: The user provides the name of the laboratory responsible for performing the benchmark experiments of interest. Selecting "All” retrieves all options simultaneously.

Geometry: The user specifies the type of geometry of interest. Selecting “Any” retrieves all options simultaneously.

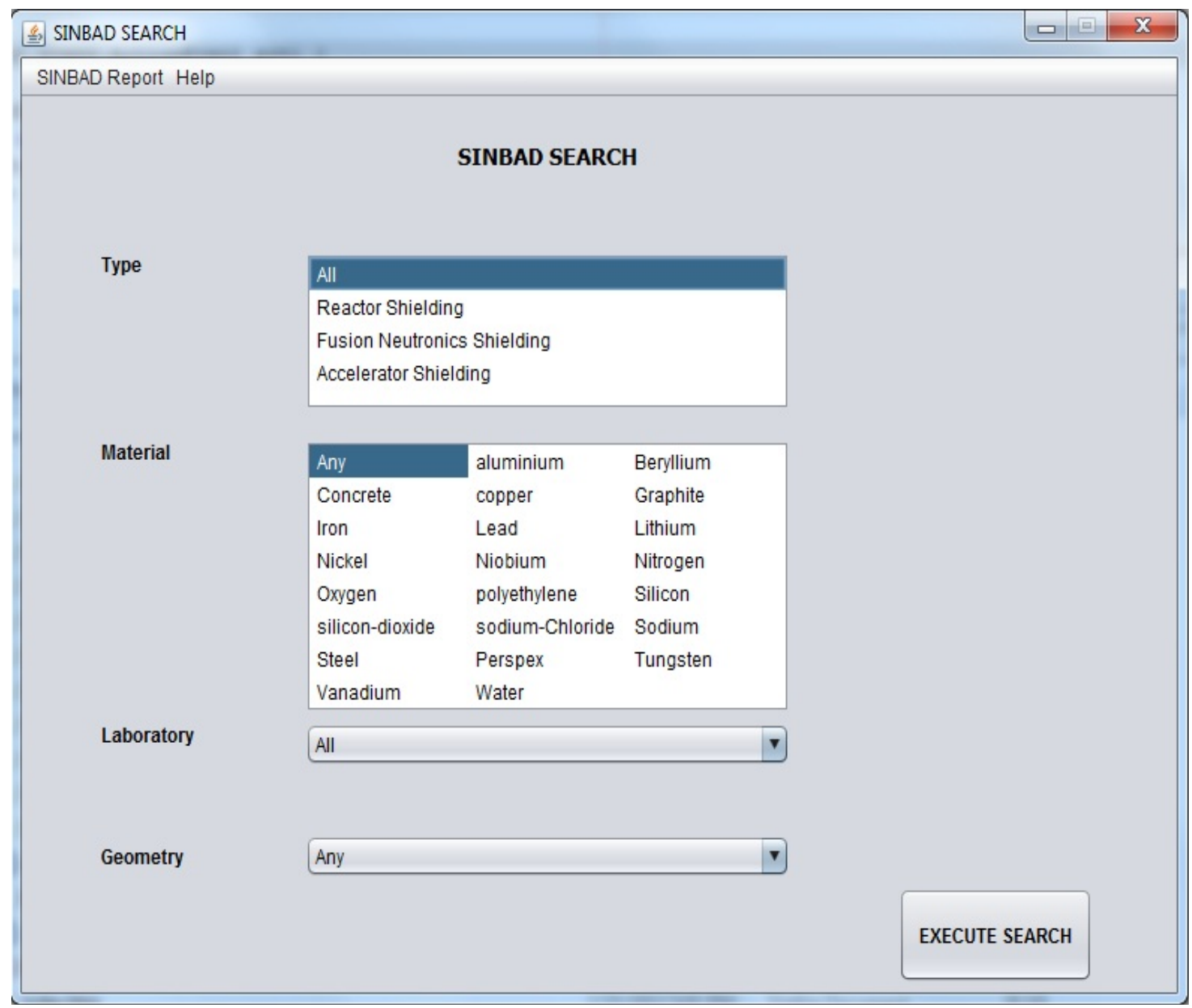

Fig. 1. Front page of the SINBAD Search Tool. 


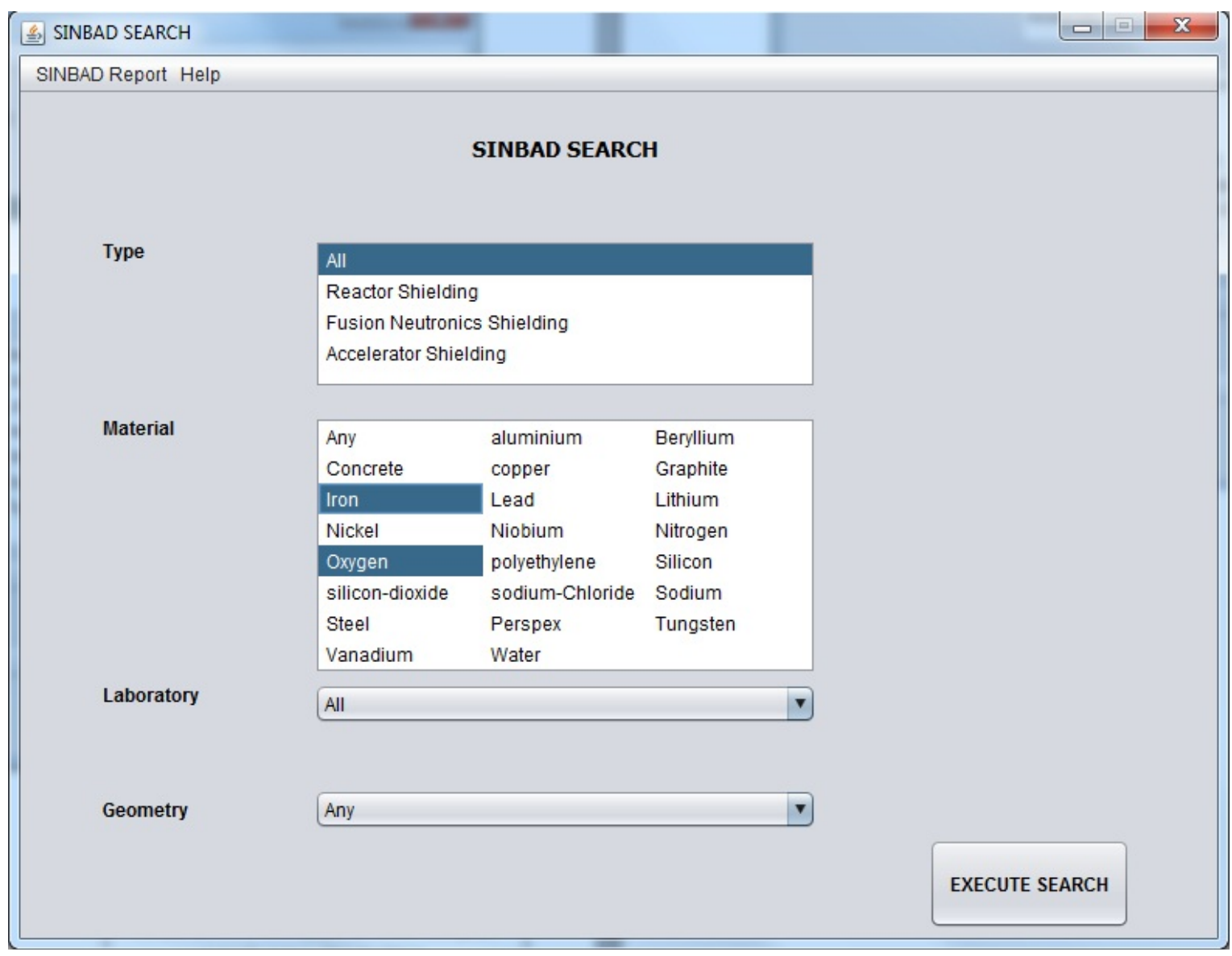

Fig. 2. Materials.

After the search parameters are defined and the "Execute Search" button is pressed, a frame that lists the benchmark names that are well matched with the user's requested choices is presented as shown in Fig. 3. 


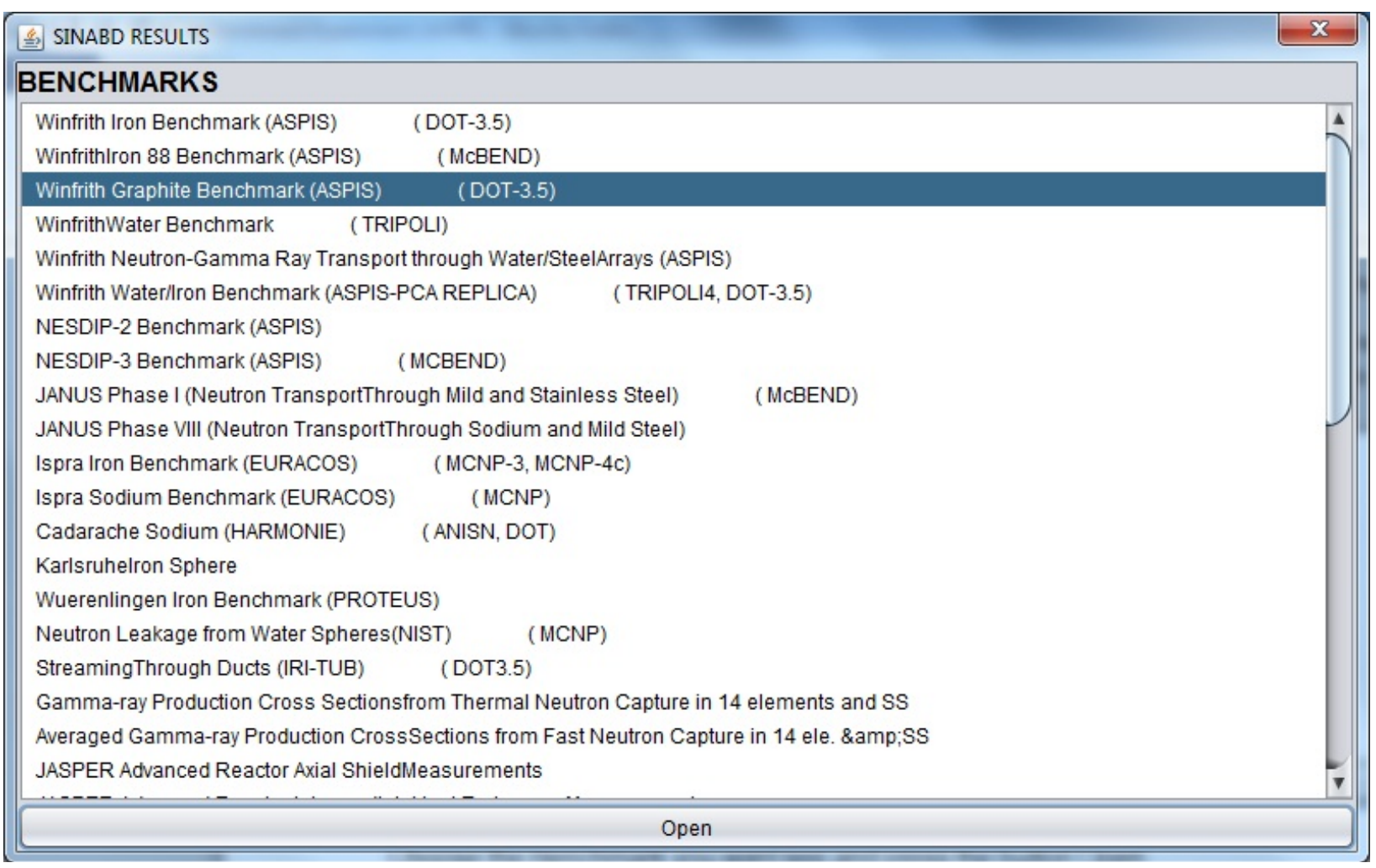

Fig. 3. Searching results.

For some benchmark experiments present in the SINBAD database, also included is an input description for some computer codes. Input data for more than one code system may exist for a single benchmark. If so, this information will be displayed in parentheses beside the benchmark name. This feature is shown in Fig. 3.

After the search is complete, the user may be able to further refine the search and choose the specific benchmark experiment needed. This is done by selecting the benchmark experiment inquired and pressing the button "Open." The benchmark abstract will open in the default browser as shown in Fig. 4.

Any available benchmark computer code input is indicated on the page with the extension .INP as shown in Fig. 5. As an example, the input to the code DOT is specified by "dot35.INP." The contents can be displayed by clicking on "dot35.INP," which will open a page in the default browser of the user's computer as shown in Fig. 6. 


\section{SINBAD ABSTRACT NEA-1517/36 Winfrith Graphite Benchmark Experiment (ASPIS)}

1. Name of Experiment:

Winfrith Graphite Benchmark Experiment (ASPIS)

2. Purpose and Phenomena Tested:

Determination of the accuracy of methods used to calculate the neutron component of nuclear heating. Threshold reaction rates were measured up to $0.7 \mathrm{~m}$ in graphite.

3. Description of the Source and Experimental Configuration:

The source is a fission plate constructed of $93 \%$ enriched uranium aluminium alloy driven by a thermal flux from the extended graphite reflector of the NESTOR reactor. The energy spectrum of the source is the one of neutrons emitted from the fission of U-235. The absolute source strength is determined by fission product counting and the spatial distribution via detailed low energy flux mapping with activation detectors.

The graphite assembly had lateral dimensions $180 \mathrm{~cm} \times 190 \mathrm{~cm}$ and total length was $177.32 \mathrm{~cm}$. It was built from graphite block of various sizes. The concrete of the approximate thickness $76 \mathrm{~cm}$ encases the whole assembly. The detectors were placed in the central block in the cylindrical plug, inserted in $6.45 \mathrm{~cm}$ radius hole along the major axis of the block.

4. Measurement System and Uncertainties:

The detectors used were:

\begin{tabular}{|c|c|c|c|c|c|}
\hline Detector & $\begin{array}{c}\text { Diameter } \\
(\mathrm{mm})\end{array}$ & $\begin{array}{l}\text { Thickness } \\
\text { (mm) }\end{array}$ & $\begin{array}{l}\text { Mass } \\
(g)\end{array}$ & $\begin{array}{l}\text { Counting } \\
\text { System }\end{array}$ & $\begin{array}{l}\text { tematic } \\
\text { or }\left(\frac{8}{6}\right)\end{array}$ \\
\hline Al-27 (n, alpha) & 50 & 3.1 & 16.72 & Ge detector & 4.0 \\
\hline$s-32(n, p)$ & 38.1 & 2.41 & 5 & Plastic scint. & 4.0 \\
\hline In-115 $\left(n, n^{\prime}\right)$ & 38 & 1.63 & 12.79 & GeLi detector & 3.0 \\
\hline $\mathrm{Rh}-103\left(\mathrm{n}, \mathrm{n}^{\prime}\right)$ & 12.7 & 0.015 & 0.20 & $\mathrm{NaI}$ & 3.0 \\
\hline
\end{tabular}

The uncertainties ( 1 sigma) may be taken as uncorrelated, and derive essentially from the absolute calibration of the counting system.

Fig. 4. Example of a benchmark page. 
9. References:

[1] M.D. Carter, P.C. Miller, A. Packwood,

The ASPIS Graphite Benchmark Experiment. Part 1 - Experimental Data and Preliminary Results, NEACRP-A-630 (1984)

[2] N. Sasamoto, K. Sakurai, A. Tsubosaka, H. Narita, M. Takemura,

K. Hayashi, Analysis of the ASPIS Graphite Benchmark Experiment with Discrete Ordinates and Monte Carlo Codes, NEACRP Specialists' Meeting on Shielding Benchmarks, Paris (1986).

[3] Alan F. Avery, AEA-RS-5628, private communication.

$\underline{[4]}$ G. A. Wright, A. Avery, M. J. Grimstone, H. F. Locke, S. Newbon, Benchmarking of the JEFF2.2 Data Library for Shielding Applications, Proceedings, 8th International Conference on Radiation Shielding, April 24-28, 1994, Arlington, Texas, U.S.A., vol.2, p.816.

10. Data and Format:

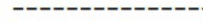

$\begin{array}{ll} & \text { Filename } \\ 1 & \frac{\text { ASC-ABS.HTM }}{\text { ASC-EXP.HTM }} \\ 3 & \frac{\text { dot35.INP }}{4} \\ 5 & \frac{\text { ASPC-1V.TIF }}{\text { ASPC-2V.TIF }} \\ 7 & \frac{\text { ASPC-3V.TIF }}{\text { ASPC-4V.TIF }}\end{array}$

\begin{aligned} Size [bytes] & Content \\ \hline 7.462 & This infor \\ 20.668 & Descriptio \\ 4.684 & Input data \\ 27.464 & Figure 1: \\ 17.362 & Figure 2: \\ 17.696 & Figure 3: \\ 16.136 & Figure 4: \\ & for the $D \\ 20.266 &$ Figure 1: \\ 12.183 & Figure 2: \\ 14.797 & Figure 3: \\ 11.482 & Figure 4: \\ & for the DO \\ 2.237 .854 & Reference \\ 458.475 & Reference \\ 789.823 & Reference \\ 578.980 & Reference \\ & \end{aligned}

Figures describing the geometry of the experiment are included in TIFF file format and GIF (preview) format.

Tables in Asc-exp.htm:

one table of axial dimensions and of chemical composition of materials

present in the configuration, 3 tables describing the source, and

4 tables of the measured reaction rates.

Fig. 5. Input link. 
IV. Input data for the DOT 3.5 calculation, using the WIMS 2 fission spectrum, for the ASPIS graphite benchmark. Taken from [1], simplified geometry as shown on Fig.4.

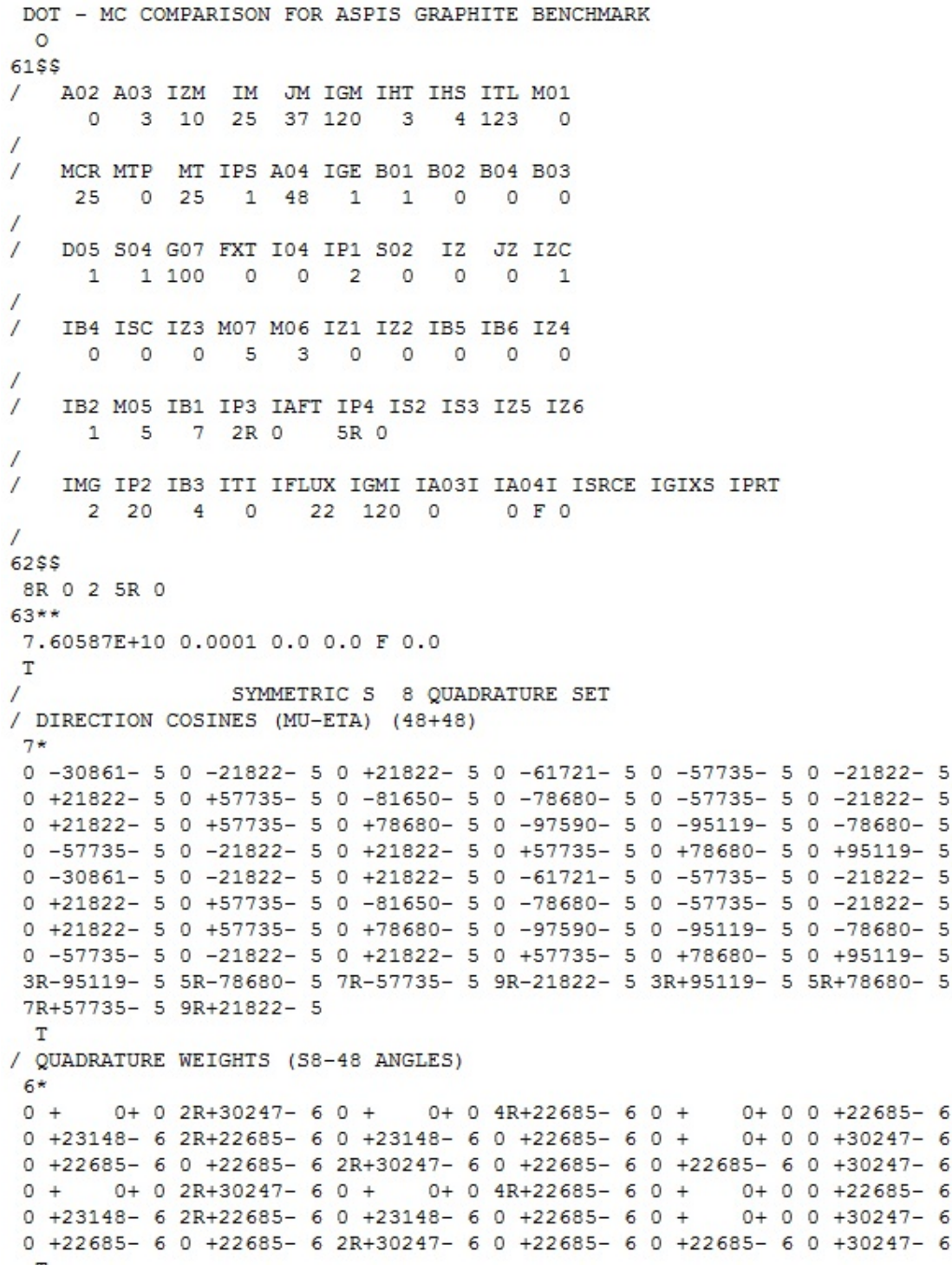

Fig. 6. Input page. 



\section{SUMMARY AND FUTURE WORK}

To facilitate searches for information in the SINBAD database, a new tool written in the Java language has been developed. This tool, called the SINBAD search tool, provides a better and efficient way to retrieve information from the SINBAD database. Searches can be performed quickly and easily. The users are no longer required to know the name of the benchmarks for which they are searching. Instead, the search is carried out by requiring information such as the facility where an experiment was done, the constituents of the experimental benchmark, and so on.

In future versions of the SINBAD search tool, features can be added to improve the program:

- $\quad$ The HTML files that contain the benchmarks abstracts could be standardized.

- An interface to run a code using the .INP files could be provided (e.g., add the ability to run MCNP code when the corresponding .INP for MCNP is given for the benchmark in the SINBAD database).

- Additional types of geometry could be added to the geometry field in the Sinbad Search interface (e.g., SLAB).

- More categories of incident particles (neutrons, electrons, and protons) could be added.

- Energy range could be added as a search criterion.

- Presently only three types of benchmark categories exist (reactor shielding, accelerator shielding, and fusion neutronic shielding), but the capability to facilitate the addition of more benchmark categories could be added without the need to reprogram the source code. 



\section{REFERENCES}

1. OECD Nuclear Energy Agency Data Bank SINBAD, http://www.nea.fr/html/science/shielding/sinbad/sinbadis.htm.

2. I. Kodeli, E. Sartori, and B. Kirk. "SINBAD Shielding Benchmark Experiments Status and Planned Activities," American Nuclear Society 14th Biennial Topical Meeting of the Radiation Protection and Shielding Division, Carlsbad, New Mexico, USA, April 3-6, 2006.

3. Radiation Safety Information Computational Center (RSICC), http://www-rsicc.ornl.gov/rsiccnew/BENCHMARKS.htm.

4. Java.sun.com.

5. NetBeans Integrated Development Environment - IDE, http://netbeans.org/index.html. 

APPENDIX A:

EXAMPLE OF THE PREVIOUS SEARCH METHOD 



\title{
SINBAD INDEX - Sorted by Shielding
}

\author{
Reactor Shielding (45) \\ Winfrith Iron Benchmark (ASPIS) \\ Winfrith Iron 88 Benchmark (ASPIS) \\ Winfrith Graphite Benchmark (ASPIS) \\ Winfrith Water/Iron Benchmark (ASPIS-PCA REPLICA) \\ Winfrith Water Benchmark \\ Winfrith Neutron-Gamma Ray Transport through Water/Steel Arrays (ASPIS) \\ NESDIP-2 Benchmark (ASPIS) \\ NESDIP-3 Benchmark (ASPIS) \\ JANUS Phase I (Neutron Transport Through Mild and Stainless Steel) \\ JANUS Phase VIII (Neutron Transport Through Sodium and Mild Steel) \\ Ispra Sodium Benchmark (EURACOS) \\ Ispra Iron Benchmark (EURACOS) \\ Cadarache Sodium (HARMONIE) \\ Karlsruhe Iron Sphere \\ Wuerenlingen Iron Benchmark (PROTEUS) \\ Neutron Leakage from Water Spheres (NIST) \\ Streaming Through Ducts (IRI-TUB) \\ Gamma-ray Production Cross Sections from Thermal Neutron Capture in 14 elements and SS \\ Averaged Gamma-ray Production Cross Sections from Fast Neutron Capture in 14 ele. \& SS \\ JASPER Advanced Reactor Axial Shield Measurements \\ JASPER Advanced Reactor Intermediate Heat Exchanger Measurements \\ JASPER Advanced Reactor Radial Shield Measurements \\ ORNL TSF Iron Broomstick \\ ORNL TSF Oxygen Broomstick \\ ORNL TSF Nitrogen Broomstick \\ ORNL TSF Sodium Broomstick \\ ORNL TSF Stainless Steel Broomstick \\ ORNL Neutron Transport Through Iron and SS - Part I \\ ORNL Neutron Transport in Thick Sodium \\ Pool Critical Assembly-Pressure Vessel Facility Benchmark \\ University of Illinois Iron Sphere (CF-252) \\ University of Tokyo-YAYOI Iron Slab \\ Radiation field parameters for pressure vessel monitoring in NRI LR-0 VVER-440 reactor \\ Radiation field parameters for pressure vessel monitoring in NRI LR-0 VVER-1000 reactor \\ Balakovo-3 VVER-1000 Ex-vessel Neutron Dosimetry Benchmark \\ VENUS-3 LWR-PVS Benchmark \\ H.B. Robinson-2 Pressure Vessel
}

Fig. A.1. Previous search tool. 
Photon Leakage Spectra from Al, Ti, Fe, $\mathrm{Cu}, \mathrm{Zr}, \mathrm{Pb}, \mathrm{U} 238$ Spheres

Photon Spectra from $\mathrm{H} 2 \mathrm{O}, \mathrm{SiO} 2$ and $\mathrm{NaCl}$

IPPE Th shell with $14 \mathrm{MeV}$ and $\mathrm{Cf}-252$ source neutrons

Baikal-1 Skyshine Benchmark Experiment

NAÏADE 1 Graphite Benchmark (60cm)

NAÏADE 1 Iron Benchmark $(60 \mathrm{~cm})$

NAÏADE 1 Light Water Benchmark $(60 \mathrm{~cm})$

SNL Polyethylene-Reflected Plutonium Metal Spheres: Subcritical Neutron and Gamma Measurements

Fusion Neutronics Shielding (29)

Nickel Sphere (OKTAVIAN)

Iron Sphere (OKTAVIAN)

Aluminium Sphere (OKTAVIAN)

Silicon Sphere (OKTAVIAN)

Tungsten Sphere (OKTAVIAN)

FNS Experimental data for fusion neutronics benchmark

FNS Integral Experiment on Graphite Cylindrical Assembly

FNS Liquid Oxygen

FNS Vanadium Cube

FNS Tungsten

FNS Skyshine

FNS Dogleg Duct Streaming

FNG-SS Shield (integral)

FNG-ITER Blanket Bulk Shield (integral)

FNG/TUD ITER Blanket Bulk Shield (spectra measurements)

FNG-ITER Neutron Streaming (integral)

FNG-ITER Dose Rate Experiment

FNG Silicon Carbide (integral)

FNG/TUD Silicon Carbide (spectra measurements)

FNG Tungsten (integral)

FNG/TUD Tungsten (spectra measurements)

TUD Iron Slab Experiment

IPPE Vanadium Shells

IPPE Iron Shells

ORNL 14-MeV Neutron SS/Borated Poly Slab

University of Illinois Iron Sphere (D-T)

$\underline{\text { KANT Spherical Beryllium Shells }}$

MEPhI empty slits streaming experiment

$\underline{\text { Juelich Li Metal Blanket Experiment }}$

Accelerator Shielding (23)

Transmission Through Shielding Materials of Neutrons and Photons Generated by $52 \mathrm{MeV}$ Protons

Transmission Through Shielding Materials of Neutrons and Photons Generated by $65 \mathrm{MeV}$ Protons

Transmission of Medium Energy Neutrons Through Concrete Shields (AVF Cyclotron)

Neutron Production from Thick Targets of Carbon, Iron, Copper, and Lead by $30-$ and $52-\mathrm{MeV}$ Protons

Fig. A.2. Previous search tool 2. 
TIARA 40 and $65 \mathrm{MeV}$ Neutron Transmission Through Iron, Concrete and Polyethylene Radioactivity induced by GeV-Protons and Spallation Neutrons using AGS accelerator Intermediate and High-Energy Accelerator Shielding Benchmarks

ROESTI I, II and III (CERN)

CERF Bonner Sphere Spectrometer Response to Charged Hadrons

CERF Radionuclide Production

CERF Residual Dose Rates

CERF Neutron Energy Spectra behind Shielding of a $120 \mathrm{GeV} / \mathrm{c}$ Hadron Beam Facility

CERN 200 and $400 \mathrm{GeV} / \mathrm{c}$ protons activation experiments

RIKEN Quasi-monoenergetic Neutron Field in 70-210 MeV Energy Range

KENS p-500 MeV shielding experiment using $4 \mathrm{~m}$ Concrete at KEK

HIMAC experiments with $\mathrm{He}, \mathrm{C}, \mathrm{Ne}, \mathrm{Ar}, \mathrm{Fe}, \mathrm{Xe}$ and $\mathrm{Si}$ ions on $\mathrm{C}, \mathrm{Al}, \mathrm{Cu} \& \mathrm{~Pb}$ targets

HIMAC High energy Neutron $(<800 \mathrm{MeV})$ Measurements in Iron

HIMAC High energy Neutron $(<800 \mathrm{MeV})$ Measurements in Concrete

BEVALAC Experiment with $\mathrm{Nb}$ Ions on $\mathrm{Nb} \& \mathrm{Al}$ Targets

MSU experiment with $\mathrm{He} \& \mathrm{C}$ ions on $\mathrm{Al}$ target

Neutron Spectra Generated by $590-\mathrm{MeV}$ Protons on a Thick $\mathrm{Pb}$ Target

ISIS Deep-Penetration Neutrons through Concrete and Iron Shields using p- $800 \mathrm{MeV}$

Simulation of the lineal energy distribution of the energy deposition in biological cells, TEPC-FLUKA Comparison

\section{SINBAD INDEX - Sorted by Directory Name}

[ $3052 \mathrm{mev}$ ] Neutron Production from Thick Targets of Carbon, Iron, Copper, and Lead by 30- and 52-MeV Protons

[52p] Transmission Through Shielding Materials of Neutrons and Photons Generated by $52 \mathrm{MeV}$ Protons

[65p] Transmission Through Shielding Materials of Neutrons and Photons Generated by $65 \mathrm{MeV}$ Protons

[ags ] Radioactivity induced by GeV-Protons and Spallation Neutrons using AGS accelerator

[asp fe ] Winfrith Iron Benchmark (ASPIS)

[ asp fe88 ] Winfrith Iron 88 Benchmark (ASPIS)

[asp grap ] Winfrith Graphite Benchmark (ASPIS)

[asp h2o ] Winfrith Water Benchmark

[asp ng] Winfrith Neutron-Gamma Ray Transport through Water/Steel Arrays (ASPIS)

[ avf75mev ] Transmission of Medium Energy Neutrons Through Concrete Shields (AVF Cyclotron)

[ balakovo ] Balakovo-3 VVER-1000 Ex-vessel Neutron Dosimetry Benchmark

[berp poly 2009 ] Polyethylene-Reflected Plutonium Metal Sphere: Subcritical Neutron and Gamma Measurements

[ bevalac ] BEVALAC Experiment with $\mathrm{Nb}$ Ions on $\mathrm{Nb} \& \mathrm{Al}$ Targets

[cerf ac5 ] CERF Radionuclide Production

[ cerf bss ] CERF Bonner Sphere Spectrometer Response to Charged Hadrons

[cerf dr3 ] CERF Residual Dose Rates

[ cerf sp ] CERF Neutron Energy Spectra behind Shielding of a $120 \mathrm{GeV} / \mathrm{c}$ Hadron Beam Facility

[cern200] CERN 200 and $400 \mathrm{GeV} / \mathrm{c}$ protons activation experiments

Fig. A.3. Previous search tool 3. 
[ eurac fe ] Ispra Iron Benchmark (EURACOS)

[eurac na ] Ispra Sodium Benchmark (EURACOS)

[FNG BLKT ] FNG-ITER Blanket Bulk Shield (integral)

[fing dose ] FNG-ITER Dose Rate Experiment

[fng str ] FNG-ITER Neutron Streaming (integral)

fng sic ] FNG Silicon Carbide (integral)

[fns ] FNS Experimental data for fusion neutronics benchmark

[FNG SS ] FNG-SS Shield (integral)

f fng w $]$ FNG Tungsten (integral)

[fns c ] FNS Integral Experiment on Graphite Cylindrical Assembly

fns duct [FNS Dogleg Duct Streaming

fns o] FNS Liquid Oxygen

[fns sky ] FNS Skyshine

[fns $\mathrm{v}$ ] FNS Vanadium Cube

fns $\mathrm{w}$ ] FNS Tungsten

harmo na Cadarache Sodium (HARMONIE)

[hbr2 ] H.B. Robinson-2 Pressure Vessel

himac ] HIMAC experiments with $\mathrm{He}, \mathrm{C}, \mathrm{Ne}, \mathrm{Ar}, \mathrm{Fe}, \mathrm{Xe}$ and $\mathrm{Si}$ ions on $\mathrm{C}, \mathrm{Al}, \mathrm{Cu} \& \mathrm{~Pb}$ targets

[ himac800fe] HIMAC High energy Neutron $(<800 \mathrm{MeV})$ Measurements in Iron

[ himac 800 conc ]HIMAC High energy Neutron $(<800 \mathrm{MeV})$ Measurements in Concrete

[iheas ] Intermediate and High-Energy Accelerator Shielding Benchmarks

[ILL FE ] University of Illinois Iron Sphere (Cf-252)

[ILL FE] University of Illinois Iron Sphere (D-T)

[ippe v ] IPPE Vanadium Shells

[ippe-fe] IPPE Iron Shells

[ippe th ] IPPE Th shell with $14 \mathrm{MeV}$ and $\mathrm{Cf}-252$ source neutrons

[iri tub ] Streaming Through Ducts (IRI-TUB)

[isis 800 ] ISIS Deep-Penetration Neutrons through Concrete and Iron Shields using p- $800 \mathrm{MeV}$

[ janus 1 ] JANUS Phase I (Neutron Transport Through Mild and Stainless Steel)

[janus 8 ] JANUS Phase VIII (Neutron Transport Through Sodium and Mild Steel)

[ JAS AX ] JASPER Advanced Reactor Axial Shield Measurements

[JAS IHX] JASPER Advanced Reactor Intermediate Heat Exchanger Measurements

[ JAS RAD ] JASPER Advanced Reactor Radial Shield Measurements

[juelich li] Juelich Li Metal Blanket Experiment

[kant ] Spherical Beryllium Shells

[kens500] KENS p-500 MeV shielding experiment using 4m Concrete at KEK

[ $\mathrm{kfk} f \mathrm{f}$ ] Karlsruhe Iron Sphere

[msu ] MSU experiment with $\mathrm{He} \& \mathrm{C}$ ions on $\mathrm{Al}$ target

[mephi] MEPhI empty slits streaming experiment

[nai60fec ] NAÏADE 1 Graphite Benchmark $(60 \mathrm{~cm})$

[nai60h2o] NAÏADE 1 Light Water Benchmark $(60 \mathrm{~cm})$

[nai60fec ] NAÏADE 1 Iron Benchmark $(60 \mathrm{~cm})$

[nesdip 2 ] NESDIP-2 Benchmark (ASPIS)

[nesdip 3 ] NESDIP-3 Benchmark (ASPIS)

[nist h2o ] Neutron Leakage from Water Spheres (NIST)

Fig. A.4. Previous search tool 4. 
[oktav al $]$ Aluminium Sphere (OKTAVIAN)

[ oktav fe ] Iron Sphere (OKTAVIAN)

[oktav ni ] Nickel Sphere (OKTAVIAN)

[oktav si] Silicon Sphere (OKTAVIAN)

[oktav w ] Tungsten Sphere (OKTAVIAN)

[PCA PV] Pool Critical Assembly-Pressure Vessel Facility Benchmark

[pca repl] Winfrith Water/Iron Benchmark (ASPIS-PCA REPLICA)

[prote fe] Wuerenlingen Iron Benchmark (PROTEUS)

[psi590 ] Neutron Spectra Generated by 590-MeV Protons on Thick Pb Target

[ rffnc ph] Photon Leakage Spectra from Al, Ti, Fe, Cu, Zr, Pb, U238 Spheres

[ffnc ph2 $]$ Photon Spectra from $\mathrm{H} 2 \mathrm{O}, \mathrm{SiO} 2$ and $\mathrm{NaCl}$

[ riken ] RIKEN Quasi-monoenergetic Neutron Field in $70-210 \mathrm{MeV}$ Energy Range

[rosti ] ROESTI I, II and III (CERN)

[SB2 GAM ] Gamma-ray Production Cross Sections from Thermal Neutron Capture in 14 elements and SS

[SB3 GAM ] Averaged Gamma-ray Production Cross Sections from Fast Neutron Capture in 14 ele. \& SS

[SB5 FUS ] ORNL 14-MeV Neutron SS/Borated Poly Slab

[SDT1 ] ORNL TSF Iron Broomstick

[SDT2 ] ORNL TSF Oxygen Broomstick

[SDT3 ] ORNL TSF Nitrogen Broomstick

[SDT4 ] ORNL TSF Sodium Broomstick

[SDT5 ] ORNL TSF Stainless Steel Broomstick

[SDT11 ] ORNL Neutron Transport Through Iron and SS - Part I

[SDT12 ] ORNL Neutron Transport in Thick Sodium

[ skyshine ] Baikal-1 Skyshine Benchmark Experiment

[tepc-fluka ] Simulation of the lineal energy distribution of the energy deposition in biological cells, TEPC-FLUKA Comparison

[tiara ] TIARA 40 and $65 \mathrm{MeV}$ Neutron Transmission Through Iron, Concrete and Polyethylene

[ tud fe ] TUD Iron Slab Experiment

[tud fng ] FNG/TUD ITER Blanket Bulk Shield (spectra measurements)

[tud sic ]FNG/TUD Silicon Carbide (spectra)

[tud w ] FNG/TUD Tungsten (spectra measurements)

[venus 3 ] VENUS-3 LWR-PVS Benchmark

[ ver440] Radiation field parameters for pressure vessel monitoring in NRI LR-0 VVER-440 reactor

[ wer1000 ] Radiation field parameters for pressure vessel monitoring in NRI LR-0 VVER-1000 reactor

[YAYOI FE] University of Tokyo-YAYOI Iron Slab

\section{SINBAD INDEX - Sorted by Laboratory}

[AGH-UST, Poland] Juelich Li Metal Blanket Experiment

[ARCS, Austria] Simulation of the lineal energy distribution of the energy deposition in biological cells, TEPC-FLUKA Comparison

[Cadarache, France - Harmonie ] Cadarache Sodium

Fig. A.5. Previous search tool 5. 
[ CEA, France ] NAÏADE 1 Graphite Benchmark $(60 \mathrm{~cm})$

[ CEA, France ] NAÏADE 1 Iron Benchmark $(60 \mathrm{~cm})$

[CEA, France ] NAÏADE 1 Light Water Benchmark $(60 \mathrm{~cm})$

[ CERN, Switzerland ] ROESTI I, II and III

[ CERN, Switzerland ] CERF Bonner Sphere Spectrometer Response to Charged Hadrons

[CERN, Switzerland ] CERF Radionuclide Production

[CERN, Switzerland ] CERF Residual Dose Rates

[ CERN, Switzerland ] CERF Neutron Energy Spectra behind Shielding of a $120 \mathrm{GeV} / \mathrm{c}$ Hadron Beam Facility

[CERN, Switzerland ] CERN 200 and $400 \mathrm{GeV} / \mathrm{c}$ protons activation experiments

[ FNS/JAEA, Japan ] FNS Experimental data for fusion neutronics benchmark

[ FNS/JAEA, Japan ] FNS Integral Experiment on Graphite Cylindrical Assembly

[FNS/JAEA, Japan ] FNS Liquid Oxygen

[FNS/JAEA, Japan ] FNS Vanadium Cube

[FNS/JAEA, Japan ] FNS Tungsten

[FNS/JAEA, Japan ] FNS Skyshine

[FNS/JAEA, Japan ] FNS Dogleg Duct Streaming

[FNG/ENEA, Italy ] FNG-SS Shield (integral meas.)

[FNG/ENEA, Italy ] FNG-ITER Blanket Bulk Shield (integral meas.)

[FNG/ENEA, Italy ] FNG-ITER Neutron Streaming (integral)

[FNG/ENEA, Italy ] FNG-ITER Dose Rate Experiment

[ FNG/ENEA. Italy ] FNG Silicon Carbide (integral)

[ FNG/ENEA, Italy ] FNG Tungsten (integral)

[ FNG/Italy, TUD/Germany ] FNG/TUD ITER Blanket Bulk Shield (spectra measurements)

[ FNG/Italy, TUD/Germany ] FNG/TUD Silicon Carbide (spectra)

[ FNG/Italy, TUD/Germany ] FNG/TUD Tungsten (spectra measurements)

[ TUD, Germany ] TUD Iron Slab Experiment

[ FzK, Germany ] Karlsruhe Iron Sphere

[FzK, Germany ] KANT Spherical Beryllium Shells

[ IPPE - FzK ] IPPE Vanadium Shells

[ IPPE, Russia] IPPE Iron Shells

[ IPPE, Russia ] IPPE Th shell with $14 \mathrm{MeV}$ and Cf-252 source neutrons

[ IRI - TUB ] Streaming Through Ducts

[ ISPRA Univ. of Pavia, Italy - EURACOS II ] Ispra Iron Benchmark

[ ISPRA Univ. of Pavia, Italy - EURACOS II ] Ispra Sodium Benchmark

[ JAEA, Japan ] TIARA 40 and $65 \mathrm{MeV}$ Neutron Transmission Through Iron, Concrete and Polyethylene

[JAEA, Japan ] Radioactivity induced by GeV-Protons and Spallation Neutrons using AGS accelerator

[JAEA, Japan ] Intermediate and High-Energy Accelerator Shielding Benchmarks

[ KEK/KENS, Japan ] KENS p-500 MeV shielding experiment using $4 \mathrm{~m}$ Concrete at KEK

[LBNL, USA ] BEVALAC Experiment with $\mathrm{Nb}$ Ions on $\mathrm{Nb} \&$ Al Targets

[MEPhI, Russian Fed. ] MEPhI empty slits streaming experiment

[MSU, USA ] Experiment with $\mathrm{He} \& \mathrm{C}$ ions on $\mathrm{Al}$ target

[NIRS, Japan ] HIMAC experiments with $\mathrm{He}, \mathrm{C}, \mathrm{Ne}, \mathrm{Ar}, \mathrm{Fe}, \mathrm{Xe}$ and $\mathrm{Si}$ ions on $\mathrm{C}, \mathrm{Al}, \mathrm{Cu} \& \mathrm{~Pb}$ targets

[ NIRS, Japan ] HIMAC High energy Neutron $(<800 \mathrm{MeV})$ Measurements in Iron

[NIRS, Japan ] HIMAC High energy Neutron $(<800 \mathrm{MeV})$ Measurements in Concrete

Fig. A.6. Previous search tool 6. 
[NIST, USA ] Neutron Leakage from Water Spheres

[NRC, USA ] H.B. Robinson-2 Pressure Vessel

[NRI, Rez ] Radiation field parameters for pressure vessel monitoring in NRI LR-0 VVER-440 reactor

[NRI, Rez ] Radiation field parameters for pressure vessel monitoring in NRI LR-0 VVER-1000 reactor

[ORNL, USA] Gamma-ray Production Cross Sections from Thermal Neutron Capture in 14 elements and SS

[ORNL, USA] Averaged Gamma-ray Production Cross Sections from Fast Neutron Capture in 14 ele. \& SS

[ORNL, USA ] JASPER Advanced Reactor Axial Shield Measurements

[ORNL, USA ] JASPER Advanced Reactor Intermediate Heat Exchanger Measurements

[ORNL, USA] JASPER Advanced Reactor Radial Shield Measurements

[ORNL, USA] ORNL TSF Iron Broomstick

[ORNL, USA] ORNL TSF Oxygen Broomstick

[ORNL, USA] ORNL TSF Nitrogen Broomstick

[ORNL, USA] ORNL TSF Sodium Broomstick

[ORNL, USA ] ORNL TSF Stainless Steel Broomstick

[ORNL, USA] ORNL Neutron Transport Through Iron and SS - Part I

[ORNL, USA ] ORNL Neutron Transport in Thick Sodium

[ORNL, USA ] Pool Critical Assembly-Pressure Vessel Facility Benchmark

[ORNL, USA] ORNL 14-MeV Neutron SS/Borated Poly Slab

[PSI, Swiss ] Neutron Spectra Generated by 590-MeV Protons on Thick Pb Target

[RAL, England] ISIS Deep-Penetration Neutrons through Concrete and Iron Shields using p- $800 \mathrm{MeV}$

[RDIPE, Russia ] Baikal-1 Skyshine Benchmark Experiment

[RFNC, Russia ] Photon Leakage Spectra from Al, Ti, Fe, Cu, Zr, Pb, U238 Spheres

[RFNC, Russia ] Photon Spectra from $\mathrm{H} 2 \mathrm{O}, \mathrm{SiO} 2$ and $\mathrm{NaCl}$

[RIKEN, Japan ] Quasi-monoenergetic Neutron Field in 70-210 MeV Energy Range

[SNL, USA] Polyethylene-Reflected Plutonium Metal Sphere HPGe

[SNL, USA] Polyethylene-Reflected Plutonium Metal Sphere NPOD

[SNL, USA] Polyethylene-Reflected Plutonium Metal Sphere SNAP

[SCK/CEN, Belguium] VENUS-3 LWR-PVS Benchmark

[SEC NRS/FZR, Russia/Germany ] Balakovo-3 VVER-1000

[University of Illinois, USA] University of Illinois Iron Sphere (D-T)

[University of Illinois, USA] University of Illinois Iron Sphere (CF-252)

[Univ. of Osaka, Japan - OKTAVIAN ] Aluminium Sphere

[Univ. of Osaka, Japan - OKTAVIAN] Iron Sphere

[Univ. of Osaka, Japan - OKTAVIAN ] Nickel Sphere

[Univ. of Osaka, Japan - OKTAVIAN] Silicon Sphere

[Univ. of Osaka, Japan - OKTAVIAN ] Tungsten Sphere

[Univ. of Osaka, Japan - AVF Cyclotron] Transmission Through Shielding Materials of Neutrons and Photons Generated by $65 \mathrm{MeV}$ Protons

[Univ. of Osaka, Japan - AVF Cyclotron] Transmission of Medium Energy Neutrons Through Concrete Shields (AVF Cyclotron)

[Univ. of Osaka and Univ. of Tokyo, Japan ] Neutron Production from Thick Targets of Carbon, Iron, Copper, and Lead by 30- and 52-MeV Protons

[Univ. of Tokyo, Japan - INS ] Transmission Through Shielding Materials of Neutrons and Photons Generated by $52 \mathrm{MeV}$ Protons

[Univ. of Tokyo, Japan - YAYOI ] University of Tokyo-YAYOI Iron Slab

[Winfrith, England - ASPIS ] Winfrith Iron Benchmark

[Winfrith, England - ASPIS ] Winfrith Iron 88 Benchmark

[Winfrith, England - ASPIS ] Winfrith Graphite Benchmark

[Winfrith, England] Winfrith Water Benchmark

Fig. A.7. Previous search tool 7. 
[ Winfirth, England - ASPIS] Winfrith Neutron-Gamma Ray Transport through Water/Steel Arrays

[ Winfrith, England - ASPIS ] JANUS Phase I (Neutron Transport Through Mild and Stainless Steel)

[ Winfrith. England - ASPIS ] JANUS Phase VIII (Neutron Transport Through Sodium and Mild Steel)

[Winfrith, England - ASPIS ] NESDIP-2 Benchmark

[ Winfrith, England - ASPIS ] NESDIP-3 Benchmark

[Winfrith, England - ASPIS ] Winfrith Water/Iron Benchmark

[ Wuerenlingen, Switzerland - PROTEUS ] Wuerenlingen Iron Benchmark

\title{
SINBAD INDEX - Sorted by Year
}

\author{
[ 1960 ] NAÏADE 1 Graphite Benchmark $(60 \mathrm{~cm})$ \\ [ 1960 ] NAÏADE 1 Iron Benchmark $(60 \mathrm{~cm})$ \\ [ 1960 ] NAÏADE 1 Light Water Benchmark $(60 \mathrm{~cm})$ \\ [1968 ] ORNL TSF Iron Broomstick \\ [1968 ] ORNL TSF Oxygen Broomstick \\ [1968 ] ORNL TSF Nitrogen Broomstick \\ [ 1968 ] ORNL TSF Sodium Broomstick \\ [1968 ] ORNL TSF Stainless Steel Broomstick \\ [ 1969 ] Gamma-ray Production Cross Sections from Thermal Neutron Capture in 14 elements and SS \\ [ 1969 ] Averaged Gamma-ray Production Cross Sections from Fast Neutron Capture in 14 ele. \& SS \\ [ 1974 ] ORNL Neutron Transport Through Iron and SS - Part I \\ [ 1974 ] ORNL Neutron Transport in Thick Sodium \\ [ 1975 ] University of Illinois Iron Sphere (CF-252) \\ [ 1975 ] University of Illinois Iron Sphere (D-T) \\ [ 1975 ] Karlsruhe Iron Sphere \\ [ 1975] Winfrith Iron Benchmark (ASPIS) \\ [ 1976 ] University of Tokyo-YAYOI Iron Slab \\ [1976-1984 ] Juelich Li Metal Blanket Experiment \\ [1978 ] Cadarache Sodium (HARMONIE) \\ [1979 ] ORNL 14-MeV Neutron SS/Borated Poly Slab \\ [1979] Neutron Spectra Generated by 590-MeV Protons on Thick Pb Target \\ [ 1980 ] Pool Critical Assembly-Pressure Vessel Facility Benchmark \\ [ 1981 Transmission Through Shielding Materials of Neutrons and Photons Generated by $52 \mathrm{MeV}$ Protons \\ [ 1982 ] Neutron Production from Thick Targets of Carbon, Iron, Copper, and Lead by 30- and 52-MeV Protons \\ [ 1983 ] CERN 200 and $400 \mathrm{GeV} / \mathrm{c}$ protons activation experiments \\ [1983-1991 ] FNS Experimental data for fusion neutronics benchmark \\ [ 1984 ] H.B. Robinson-2 Pressure Vessel \\ [ 1984 ] Winfrith Graphite Benchmark (ASPIS) \\ [ 1984 ] Winfrith Water Benchmark \\ [1984 ] Winfrith Water/Iron Benchmark (ASPIS-PCA REPLICA)
}

Fig. A.8. Previous search tool 8 . 


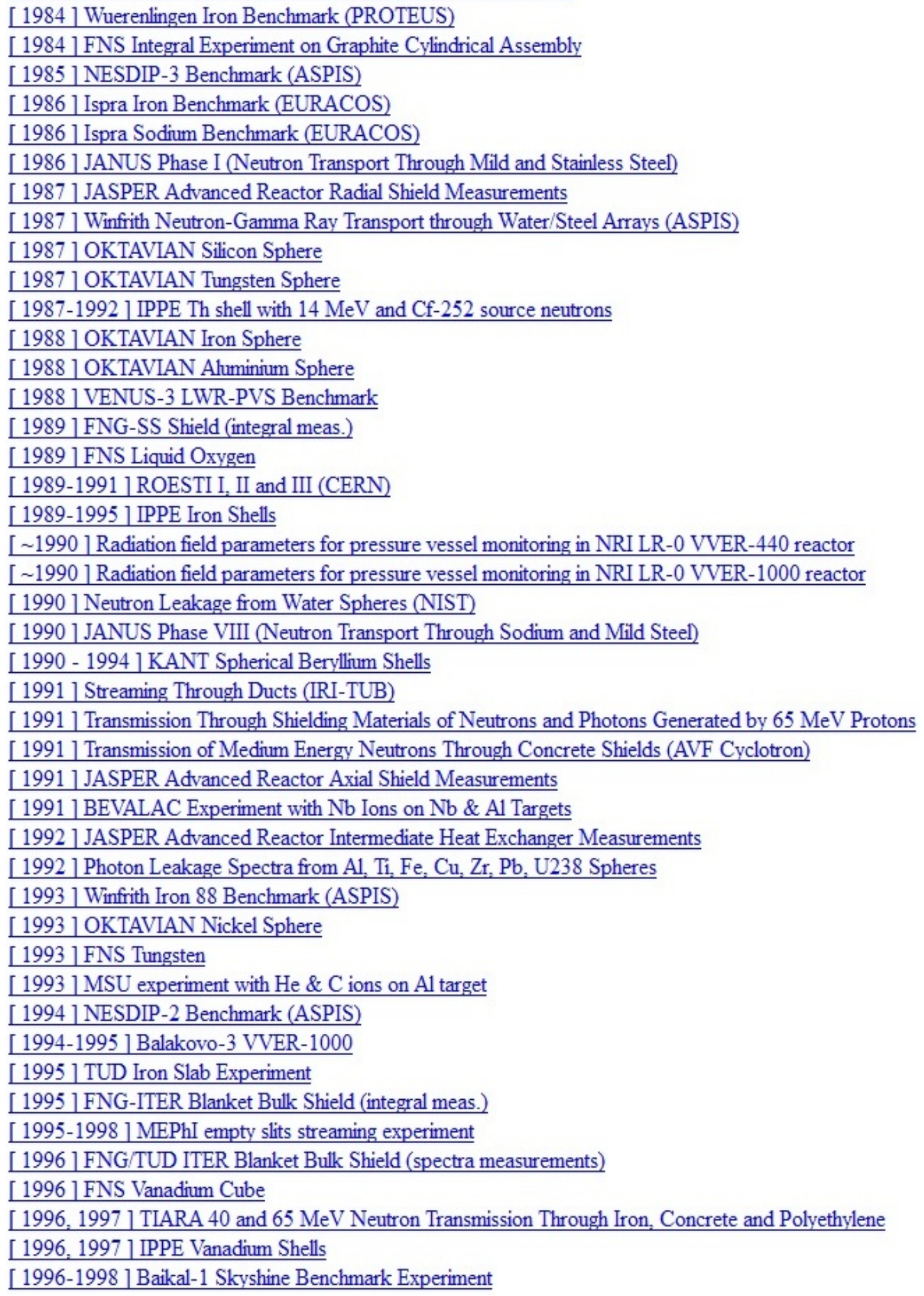

Fig. A.9. Previous search tool 9. 
[1997-1998 ] FNG-ITER Neutron Streaming (integral)

[ 1997 - 1999 ] HIMAC experiments with $\mathrm{He}, \mathrm{C}, \mathrm{Ne}, \mathrm{Ar}, \mathrm{Fe}, \mathrm{Xe}$ and $\mathrm{Si}$ ions on C, Al, Cu \& Pb targets

1998 ] ISIS Deep-Penetration Neutrons through Concrete and Iron Shields using p- $800 \mathrm{MeV}$

[ 1998? ] RIKEN Quasi-monoenergetic Neutron Field in 70-210 MeV Energy Range

[2000-2001 ] FNG-ITER Dose Rate Experiment

2001 ] FNG Silicon Carbide (integral)

2001 ] FNG/TUD Silicon Carbide (spectra measurements)

[2001] HIMAC High energy Neutron $(<800 \mathrm{MeV})$ Measurements in Concrete

2001 Radioactivity induced by GeV-Protons and Spallation Neutrons using AGS accelerator

2002 ] FNG Tungsten (integral)

[2002 ] FNG/TUD Tungsten (spectra measurements)

2002 ] FNS Skyshine

2002 ] HIMAC High energy Neutron $(<800 \mathrm{MeV})$ Measurements in Iron

[2002-2004 ] KENS p-500 MeV shielding experiment using 4m Concrete at KEK

[ 2003 ] CERF Bonner Sphere Spectrometer Response to Charged Hadrons

2003 ] CERF Residual Dose Rates

2003 ] Simulation of the lineal energy distribution of the energy deposition in biological cells. TEPC-FLUKA Comparison

[2003 ] CERF Radionuclide Production

[2004 ] FNS Dogleg Duct Streaming

2004 ] CERF Neutron Energy Spectra behind Shielding of a $120 \mathrm{GeV} / \mathrm{c}$ Hadron Beam Facility

2006 ] Photon Spectra from $\mathrm{H} 2 \mathrm{O}, \mathrm{SiO} 2$ and $\mathrm{NaCl}$

[2009 ] Polyethylene-Reflected Plutonium Metal Sphere: Subcritical Neutron and Gamma Measurements

[?] Intermediate and High-Energy Accelerator Shielding Benchmarks

\section{SINBAD INDEX - Sorted by Material}

[Air ] Streaming Through Ducts (IRI-TUB)

[Air ] FNG-ITER Neutron Streaming (integral)

[Air ] FNS Dogleg Duct Streaming

[Air ] FNS Skyshine

[Air ] Baikal-1 Skyshine Benchmark Experiment

[Air ] RIKEN Quasi-monoenergetic Neutron Field in 70-210 MeV Energy Range

[Air ] Simulation of the lineal energy distribution of the energy deposition in biological cells, TEPC-FLUKA Comparison

[Air ] CERF Bonner Sphere Spectrometer Response to Charged Hadrons

[Aluminium ] Aluminium Sphere (OKTAVIAN)

[Air ] MEPhI empty slits streaming experiment

[Aluminium ] MSU experiment with $\mathrm{He} \& \mathrm{C}$ ions on $\mathrm{Al}$ target

[Beryllium ] KANT Spherical Beryllium Shells

Concrete ] HIMAC High energy Neutron $(<800 \mathrm{MeV})$ Measurements in Concrete

[Concrete ] KENS p-500 MeV shielding experiment using $4 \mathrm{~m}$ Concrete at KEK

[Concrete ] Transmission of Medium Energy Neutrons Through Concrete Shields (AVF Cyclotron)

Fig. A.10. Previous search tool 10. 
[ Copper ] CERN 200 and $400 \mathrm{GeV} / \mathrm{c}$ protons activation experiments

[ Copper ] CERF Neutron Energy Spectra behind Shielding of a $120 \mathrm{GeV} / \mathrm{c}$ Hadron Beam Facility

[ Graphite ] NAÏADE 1 Graphite Benchmark $(60 \mathrm{~cm})$

[Graphite] Winfrith Graphite Benchmark (ASPIS)

[ Graphite ] FNS Integral Experiment on Graphite Cylindrical Assembly

[ Iron ] NAÏADE 1 Iron Benchmark $(60 \mathrm{~cm})$

[ Iron ] Karlsruhe Iron Sphere

[ron ] Winfrith Iron Benchmark (ASPIS)

[ Iron] Wuerenlingen Iron Benchmark (PROTEUS)

[ Iron ] Ispra Iron Benchmark (EURACOS)

[ Iron] Iron Sphere (OKTAVIAN)

[ Iron] Winfrith Iron 88 Benchmark (ASPIS)

[ Iron ] Iron Slab Experiment (TUD)

[ Iron ] University of Tokyo-YAYOI Iron Slab

[ Iron ] ORNL TSF Iron Broomstick

[ Iron ] University of Illinois Iron Sphere (CF-252)

[ Iron ] University of Illinois Iron Sphere (D-T)

[ Iron ] IPPE Iron Shells

[ Iron ] HIMAC High energy Neutron $(<800 \mathrm{MeV})$ Measurements in Iron

[ Iron, Concrete ] ISIS Deep-Penetration Neutrons through Concrete and Iron Shields using p- $800 \mathrm{MeV}$

[ Iron, Concrete, Polyethylene ] TIARA 40 and $65 \mathrm{MeV}$ Neutron Transmission Through Iron, Concrete and Polyethylene [ Iron, Lead ] ROESTI I, II and III (CERN)

[Lead ] Neutron Spectra Generated by $590-\mathrm{MeV}$ Protons on Thick Pb Target

[ Lithium ] Juelich Li Metal Blanket Experiment

[Nickel] Nickel Sphere (OKTAVIAN)

[Niobium. Aluminum ] BEVALAC Experiment with $\mathrm{Nb}$ Ions on $\mathrm{Nb} \& \mathrm{Al}$ Targets

[Nitrogen] ORNL TSF Nitrogen Broomstick

[Oxygen ] FNS Liquid Oxygen

[Oxygen ] ORNL TSF Oxygen Broomstick

[Plutonium ] Polyethylene-Reflected Plutonium Metal Sphere: Subcritical Neutron and Gamma Measurements HPGe

[Plutonium ] Polyethylene-Reflected Plutonium Metal Sphere: Subcritical Neutron and Gamma Measurements NPOD

[Plutonium ] Polyethylene-Reflected Plutonium Metal Sphere: Subcritical Neutron and Gamma Measurements SNAP

[Silicon] Silicon Sphere (OKTAVIAN)

[Silicon Carbide ] FNG Silicon Carbide (integral)

[Silicon Carbide ] FNG/TUD Silicon Carbide (spectra)

[Sodium ] Cadarache Sodium (HARMONIE)

[Sodium ] Ispra Sodium Benchmark (EURACOS)

[Sodium ] ORNL TSF Sodium Broomstick

[Sodium ] ORNL Neutron Transport in Thick Sodium

[ Sodium. Steel ] JANUS Phase VIII (Neutron Transport Through Sodium and Mild Steel)

[ Steel ] JANUS Phase I (Neutron Transport Through Mild and Stainless Steel)

[Stainless Steel ] ORNL TSF Stainless Steel Broomstick

[ Stainless Steel ] FNG-SS Shield (integral meas.)

[ Iron and Stainless Steel ] ORNL Neutron Transport Through Iron and SS - Part I

Fig. A.11. Previous search tool 11. 
[Stainless Steel and Poly ] ORNL 14-MeV Neutron SS/Borated Poly Slab

[ Stainless Steel, Poly, Copper ] FNG-ITER Blanket Bulk Shield (integral)

[Stainless Steel, Poly, Copper ] FNG/TUD ITER Blanket Bulk Shield (spectra)

[ Stainless Steel, Perspex ] FNG-ITER Dose Rate Experiment

[Thorium ] IPPE Th shell with $14 \mathrm{MeV}$ and Cf-252 source neutrons

[ Tungsten ] Tungsten Sphere (OKTAVIAN)

[ Tungsten ] FNS Tungsten

[ Tungsten ] FNG Tungsten (integral)

[ Tungsten ] FNG/TUD Tungsten (spectra)

[Vanadium ] FNS Vanadium Cube

[Vanadium ] IPPE Vanadium Shells

[ Water ] Winfrith Water Benchmark

[ Water ] Neutron Leakage from Water Spheres (NIST)

[Water ] NAÏADE 1 Light Water Benchmark $(60 \mathrm{~cm})$

[Water, Graphite, Iron ] Transmission Through Shielding Materials of Neutrons and Photons Generated by $52 \mathrm{MeV}$ Protons

[Water, Graphite, Lead] Transmission Through Shielding Materials of Neutrons and Photons Generated by $65 \mathrm{MeV}$ Protons

[ Water, Iron] Pool Critical Assembly-Pressure Vessel Facility Benchmark

[ Water, Iron ] Winfrith Water/Iron Benchmark (ASPIS-PCA REPLICA)

[Water, Steel] Winfrith Neutron-Gamma Ray Transport through Water/Steel Arrays (ASPIS)

[Water, Steel, Aluminium ] NESDIP-3 Benchmark (ASPIS)

[Water, Steel, Aluminium ] NESDIP-2 Benchmark (ASPIS)

[Water, Silicon-Dioxide, Sodium-Chloride ] Photon Spectra from $\mathrm{H} 2 \mathrm{O}, \mathrm{SiO} 2$ and $\mathrm{NaCl}$

MULTIPLE (MORE THAN 3 Materials, either INTEGRAL or Separate)

Radiation field parameters for pressure vessel monitoring in NRI LR-0 VVER-440 reactor

Radiation field parameters for pressure vessel monitoring in NRI LR-0 VVER-1000 reactor

Balakovo-3 VVER-1000 Ex-vessel Neutron Dosimetry Benchmark

CERF Residual Dose Rates

CERF Radionuclide Production

H.B. Robinson-2 Pressure Vessel

JASPER Advanced Reactor Axial Shield Measurements

JASPER Advanced Reactor Intermediate Heat Exchanger Measurements

JASPER Advanced Reactor Radial Shield Measurements

Gamma-ray Production Cross Sections from Thermal Neutron Capture in 14 elements and SS

Averaged Gamma-ray Production Cross Sections from Fast Neutron Capture in 14 ele. \& SS

HIMAC experiments with $\mathrm{He}, \mathrm{C}, \mathrm{Ne}, \mathrm{Ar}, \mathrm{Fe}, \mathrm{Xe}$ and $\mathrm{Si}$ ions on $\mathrm{C}, \mathrm{Al}, \mathrm{Cu} \& \mathrm{~Pb}$ targets

VENUS-3 LWR-PVS Benchmark

Photon Leakage Spectra from Al, Ti, Fe, Cu, Zr, Pb, U238 Spheres

Neutron Production from Thick Targets of Carbon, Iron, Copper, and Lead by 30- and 52-MeV Protons

Radioactivity induced by GeV-Protons and Spallation Neutrons using AGS accelerator

FNS Experimental data for fusion neutronics benchmark

Intermediate and High-Energy Accelerator Shielding Benchmarks

Fig. A.12. Previous search tool 12. 
APPENDIX B:

LIST OF THE SINBAD SEARCH TOOL CODE, WRITTEN IN JAVA LANGUAGE ON THE NETBEANS IDE 7.1 PLATFORM 



\section{SinbadInterface.java (The Main Window)}

import java.io.FileNotFoundException;

import java.io.IOException;

import java.util.ArrayList;

import java.util.Arrays;

import java.util.Iterator;

import java.util.List;

import java.util.logging.Level;

import java.util.logging.Logger;

import javax.swing.JOptionPane;

public class SinbadInterface extends javax.swing.JFrame \{ public ArrayList keylist= new ArrayList();

String typ="All";

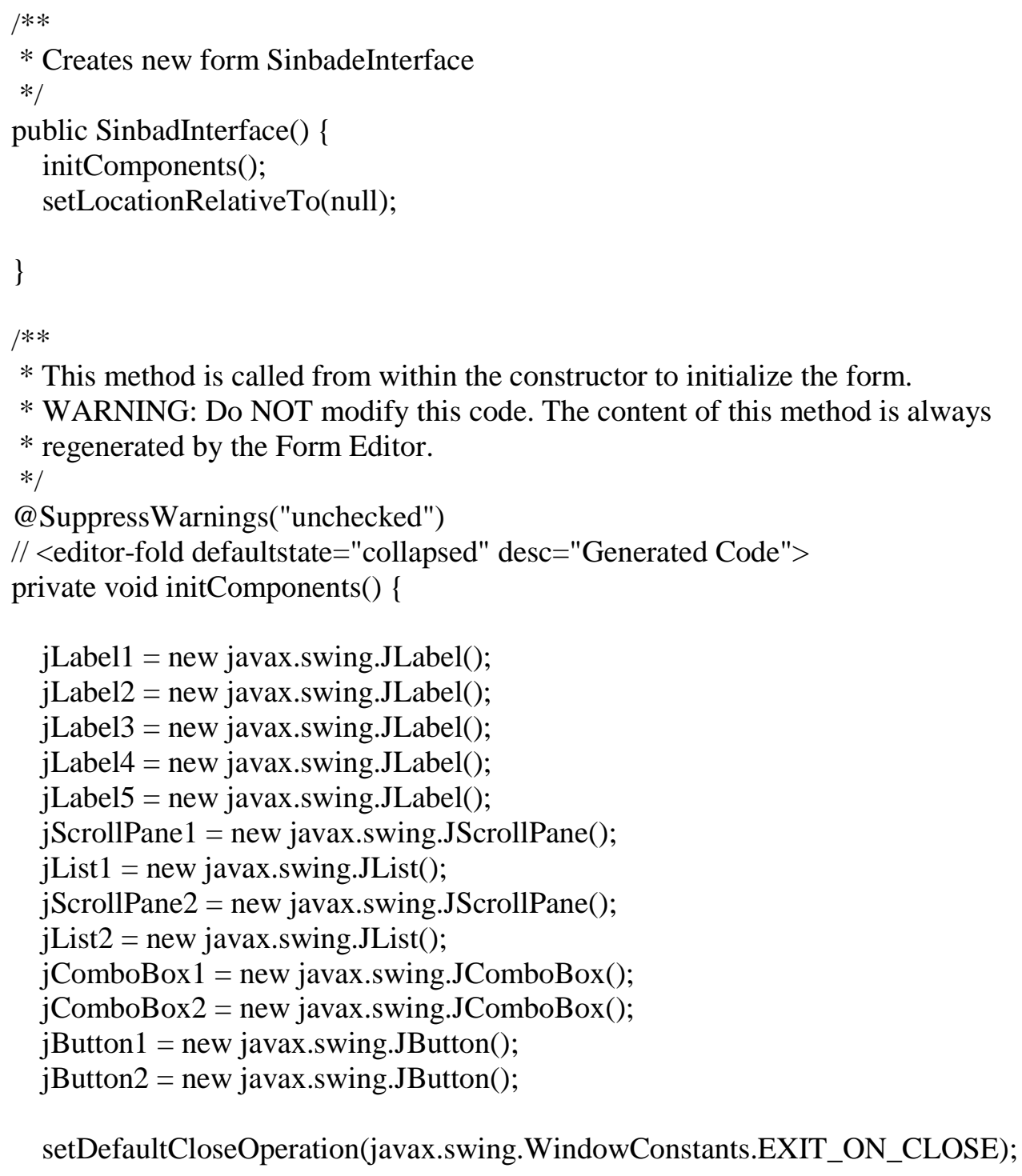


setTitle("SINBAD SEARCH");

setResizable(false);

jLabel1.setFont(new java.awt.Font("Tahoma", 1, 14)); // NOI18N

jLabel1.setText("SINBAD SEARCH");

jLabel2.setFont(new java.awt.Font("Arial", 1, 12)); // NOI18N

jLabel2.setText("Type");

jLabel3.setFont(new java.awt.Font("Arial", 1, 12)); // NOI18N

jLabel3.setText("Material");

jLabel4.setFont(new java.awt.Font("Arial", 1, 12)); // NOI18N

jLabel4.setText("Laboratory");

jLabel5.setFont(new java.awt.Font("Arial", 1, 12)); // NOI18N

jLabel5.setText("Geometry");

jList1.setModel(new javax.swing.AbstractListModel() \{

String[] strings = \{ "All", "Reactor Shielding", "Fusion Neutronics Shielding", "Accelerator

Shielding" \};

public int getSize() \{ return strings.length; \}

\}$)$;

public Object getElementAt(int i) \{ return strings[i]; \}

jList1.setSelectionMode(javax.swing.ListSelectionModel.SINGLE_SELECTION);

jList1.setToolTipText("');

jList1.setSelectedIndex(0);

jList1.addListSelectionListener(new javax.swing.event.ListSelectionListener() \{

public void valueChanged(javax.swing.event.ListSelectionEvent evt) \{

type(evt);

\}

\}$)$;

jScrollPane1.setViewportView(jList1);

jList2.setModel(new javax.swing.AbstractListModel() \{

String[] strings = \{ "Any", "aluminium ", "Beryllium ", "Concrete", "copper ", "Graphite ", "Iron

", "Lead ", "Lithium ", "Nickel ", "Niobium ", "Nitrogen ", "Oxygen", "polyethylene ", "Silicon ",

"silicon-dioxide ", "sodium-Chloride ", "Sodium ", "Steel ", "Perspex ", "Tungsten ", "Vanadium ",

"Water" \};

public int getSize() \{ return strings.length; \}

public Object getElementAt(int i) \{ return strings[i]; \}

\}$)$;

jList2.setLayoutOrientation(javax.swing.JList.HORIZONTAL_WRAP);

jList2.setSelectedIndex(0);

jScrollPane2.setViewportView(jList2);

jComboBox1.setModel(new javax.swing.DefaultComboBoxModel(new String[] \{ "All", "AGHUST/Poland", "ARCS/Austria", "Cadarache/France-Harmonie", "CEA/France", "CERN/Switzerland", "FNS/JAEA-Japan", "FNG/ENEA-Italy", "FNG/Italy-TUD/Germany", "TUD/Germany", "FzK/Germany", "IPPE/Russia", "IRI-TUB", "ISPRA Univ. of Pavia/Italy-EURACOS II", "JAEA/Japan", "KEK/KENS-Japan", "LBNL/USA", "MEPhI/Russian Fed.", "MSU/USA", 
"NIRS/Japan", "NIST/USA", "NRC/USA", "NRI/Rez", "ORNL/USA", "PSI/Swiss", "RAL/England", "RDIPE/Russia", "RFNC/Russia", "RIKEN/Japan", "SNL/USA", "SCK/CEN-Belgium", "SEC

NRS/FZR-Russia/Germany", "University of Illinois/USA", "Univ. of Osaka/Japan-OKTAVIAN", "Univ. of Osaka/Japan-AVF Cyclotron", "Univ. of Osaka and Univ. of Tokyo/Japan", "Univ. of Tokyo/JapanINS", "Univ. of Tokyo/Japan-YAYOI", "Winfrith/England-ASPIS", "Wuerenlingen/SwitzerlandPROTEUS" \}));

jComboBox2.setModel(new javax.swing.DefaultComboBoxModel(new String[] \{ "Any", "Sphere", "Cylindrical" \}));

jButton1.setFont(new java.awt.Font("Arial", 1, 12)); // NOI18N

jButton1.setText("EXECUTE SEARCH");

jButton1.addActionListener(new java.awt.event.ActionListener() \{

public void actionPerformed(java.awt.event.ActionEvent evt) \{

\} search(evt);

\}$)$;

jButton2.setText("jButton2");

javax.swing.GroupLayout layout = new javax.swing.GroupLayout(getContentPane());

getContentPane().setLayout(layout);

layout.setHorizontalGroup(

layout.createParallelGroup(javax.swing.GroupLayout.Alignment.LEADING)

.addGroup(layout.createSequentialGroup()

.addGap(53, 53, 53)

.addGroup(layout.createParallelGroup(javax.swing.GroupLayout.Alignment.LEADING) .addComponent(jLabel4)

.addComponent(jLabel3)

.addComponent(jLabel2)

.addComponent(jLabel5))

.addGap(74, 74, 74)

.addGroup(layout.createParallelGroup(javax.swing.GroupLayout.Alignment.CENTER)

.addComponent(jLabel1)

.addComponent(jScrollPane1)

.addComponent(jScrollPane2)

.addComponent(jComboBox1, 0, javax.swing.GroupLayout.DEFAULT_SIZE,

Short.MAX_VALUE)

.addComponent(jComboBox2, 0, javax.swing.GroupLayout.DEFAULT_SIZE,

Short.MAX_VALUE))

.addContainerGap(258, Short.MAX_VALUE))

.addGroup(javax.swing.GroupLayout.Alignment.TRAILING, layout.createSequentialGroup() .addContainerGap(javax.swing.GroupLayout.DEFAULT_SIZE, Short.MAX_VALUE) .addComponent(jButton1)

); .addGap(56, 56, 56))

layout.setVerticalGroup( layout.createParallelGroup(javax.swing.GroupLayout.Alignment.LEADING) .addGroup(layout.createSequentialGroup() .addGap(28, 28, 28)

.addComponent(jLabel1) 


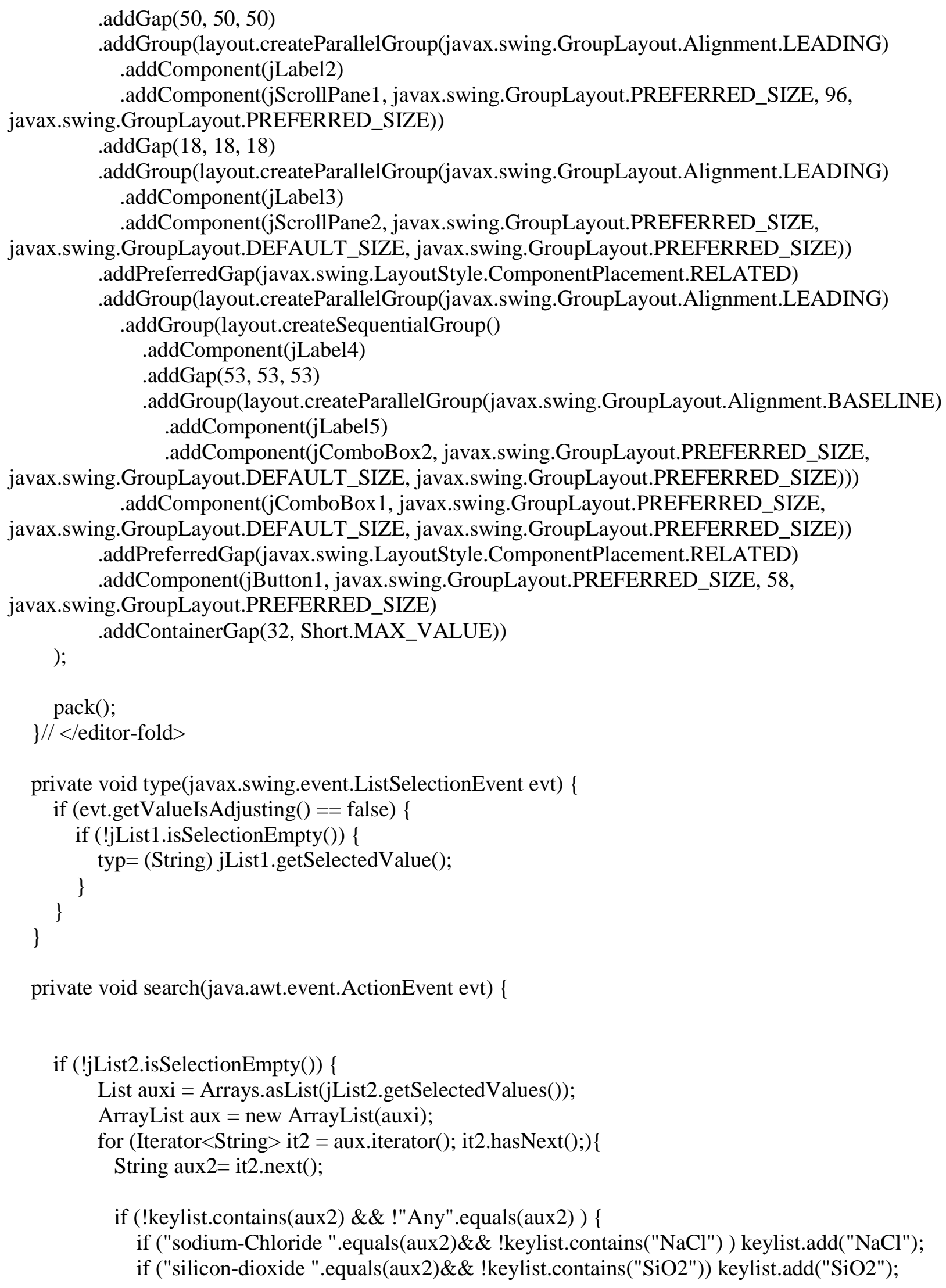

private void search(java.awt.event.ActionEvent evt) \{

if (!jList2.isSelectionEmpty()) \{

List auxi $=$ Arrays.asList(jList2.getSelectedValues());

ArrayList aux = new ArrayList(auxi);

for (Iterator $<$ String $>$ it2 = aux.iterator(); it2.hasNext(); \{

String aux2= it2.next();

if (!keylist.contains(aux2) \&\& !"Any".equals(aux2) ) \{

if ("sodium-Chloride ".equals(aux2)\&\& !keylist.contains("NaCl") ) keylist.add("NaCl"); if ("silicon-dioxide ".equals(aux2)\&\& !keylist.contains("SiO2")) keylist.add("SiO2"); 

keylist.add(aux2);

if (!"sodium-Chloride ".equals(aux2) \&\& !"silicon-dioxide ".equals(aux2) )

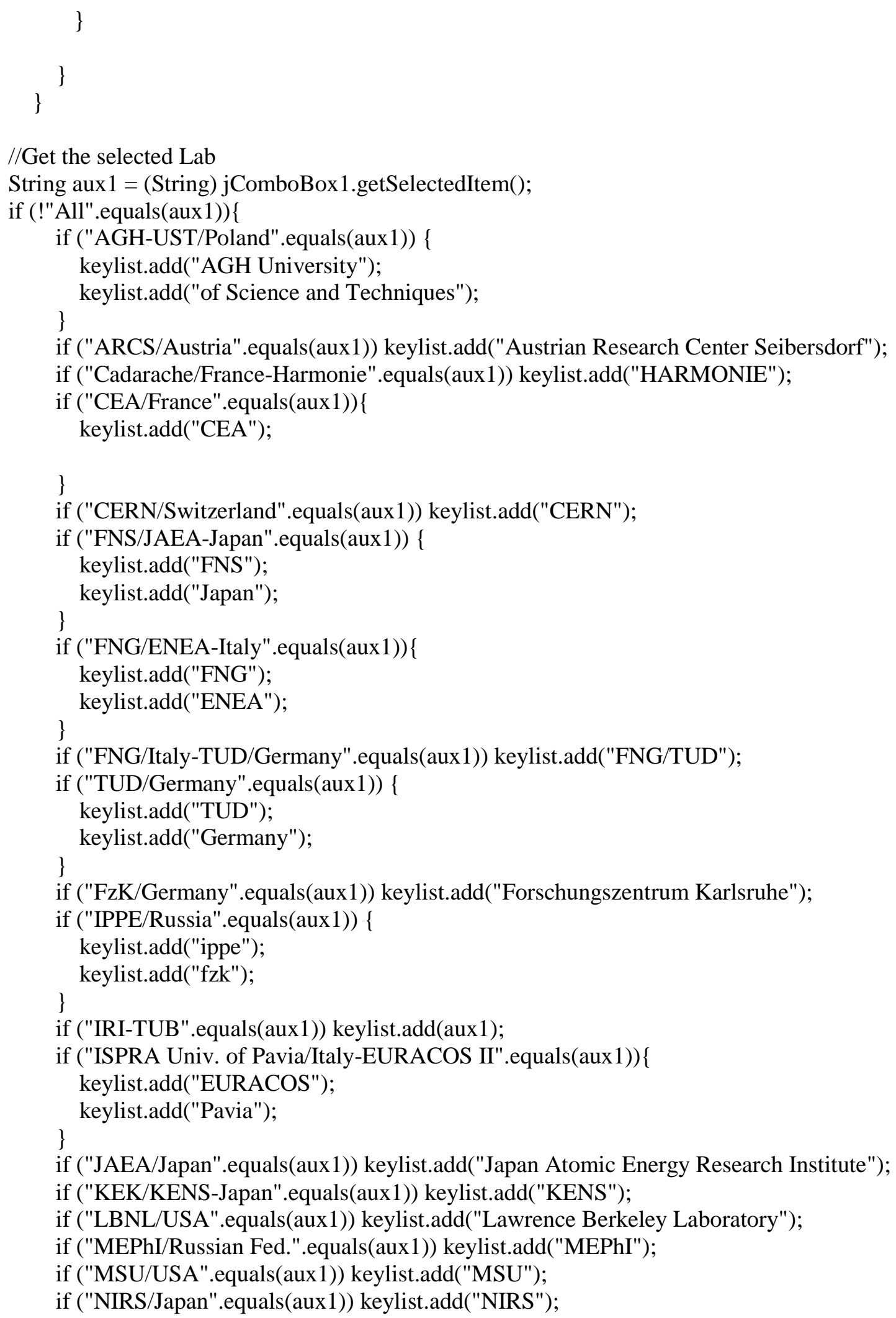




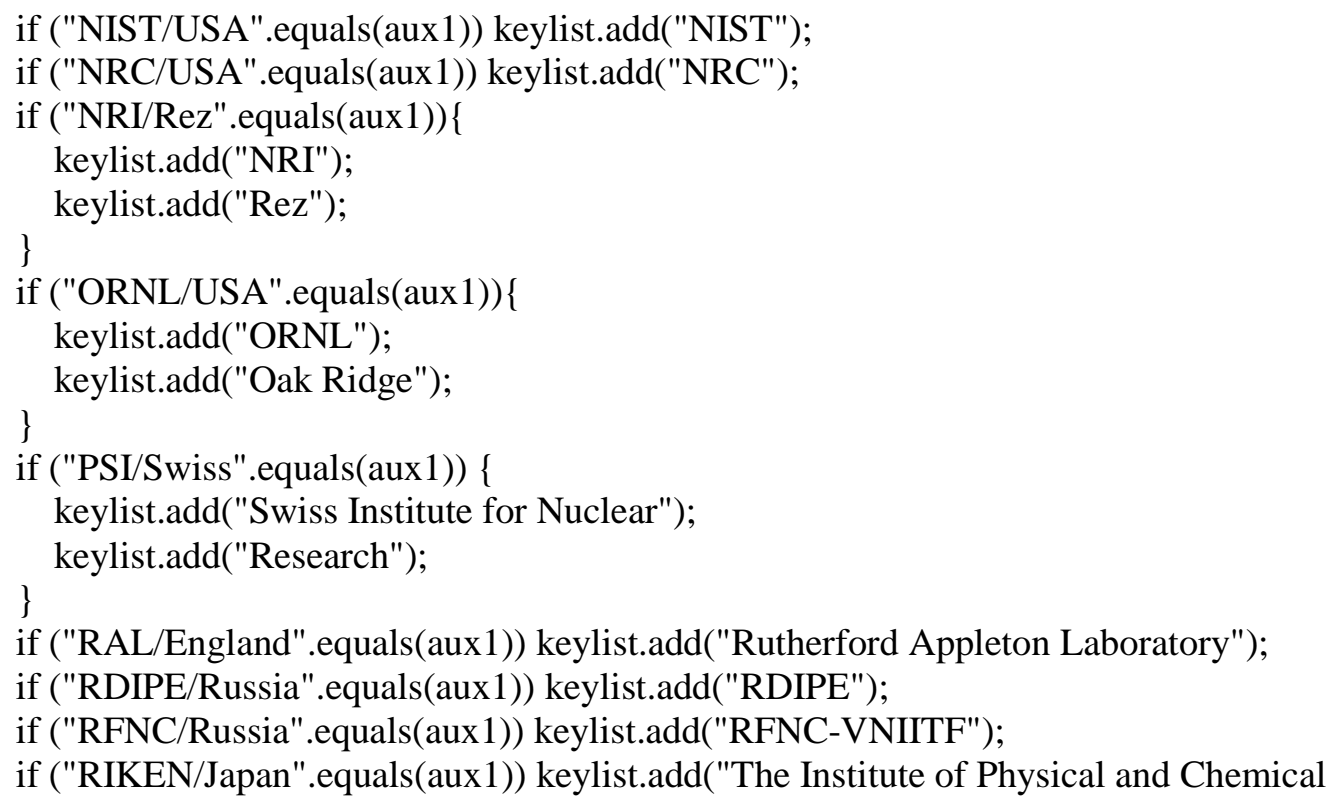


if ("Cylindrical".equals(aux2)) keylist.add(aux2);

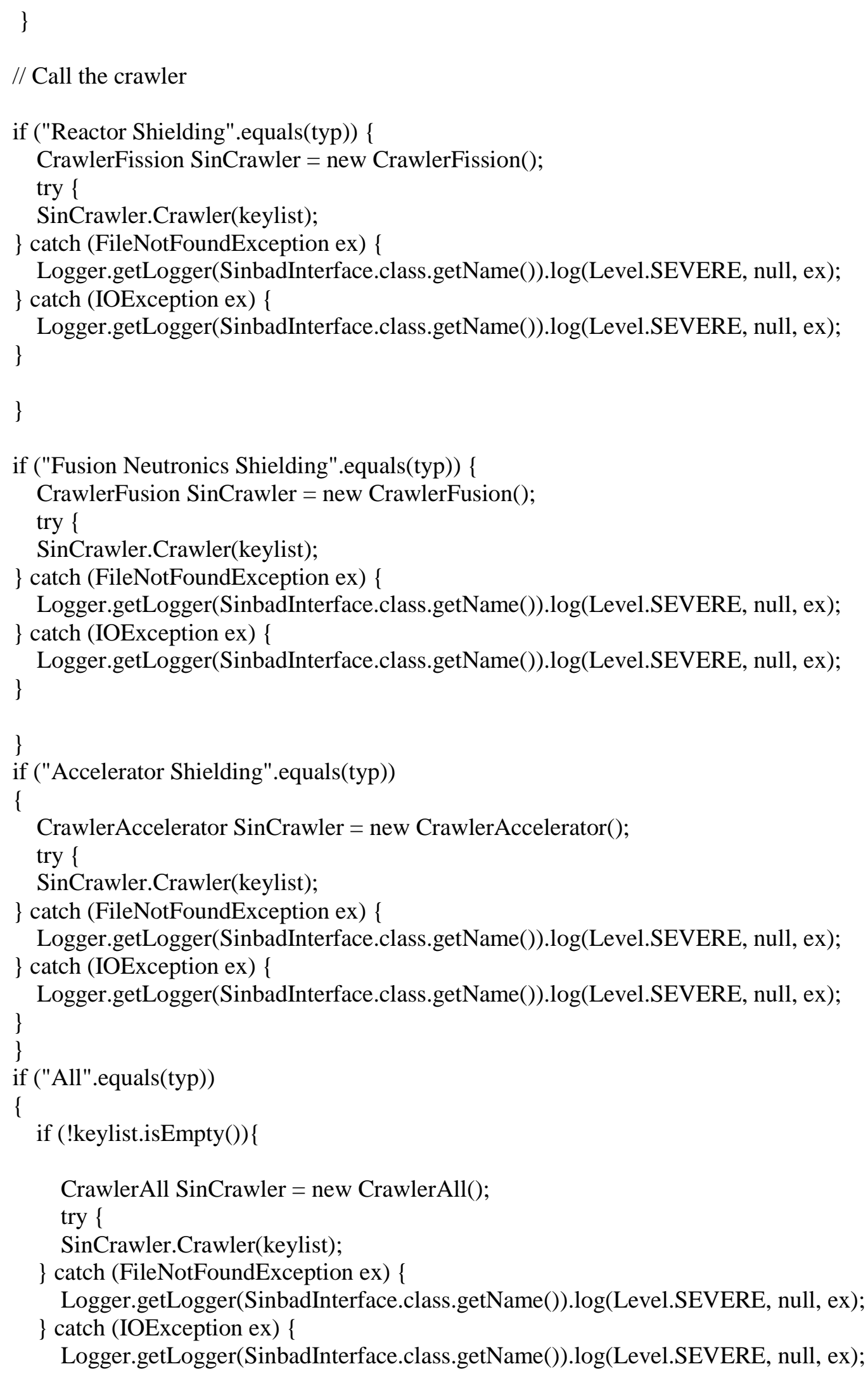


\}else JOptionPane.showMessageDialog(null,"You need to set parameters for the search");

\}

keylist.clear();

jList1.setSelectedIndex(0);

jList2.setSelectedIndex(0);

jComboBox1.setSelectedIndex(0);

jComboBox2.setSelectedIndex(0);

\}

/**

* @param args the command line arguments

*/

public static void main(String args[]) \{

/*

* Set the Nimbus look and feel

$* /$

$/ /<$ editor-fold defaultstate="collapsed" desc=" Look and feel setting code (optional) ">

/*

* If Nimbus (introduced in Java SE 6) is not available, stay with the

* default look and feel. For details see

* http://download.oracle.com/javase/tutorial/uiswing/lookandfeel/plaf.html

$*$ /

try \{

for (javax.swing.UIManager.LookAndFeelInfo info :

javax.swing.UIManager.getInstalledLookAndFeels()) \{

if ("Nimbus".equals(info.getName())) \{ javax.swing.UIManager.setLookAndFeel(info.getClassName()); break;

\} catch (ClassNotFoundException ex) \{

java.util.logging.Logger.getLogger(SinbadInterface.class.getName()).log(java.util.logging.Level.SEVER E, null, ex);

\} catch (InstantiationException ex) \{

java.util.logging.Logger.getLogger(SinbadInterface.class.getName()).log(java.util.logging.Level.SEVER E, null, ex);

\} catch (IllegalAccessException ex) \{

java.util.logging.Logger.getLogger(SinbadInterface.class.getName()).log(java.util.logging.Level.SEVER E, null, ex);

\} catch (javax.swing.UnsupportedLookAndFeelException ex) \{

java.util.logging.Logger.getLogger(SinbadInterface.class.getName()).log(java.util.logging.Level.SEVER E, null, ex); 


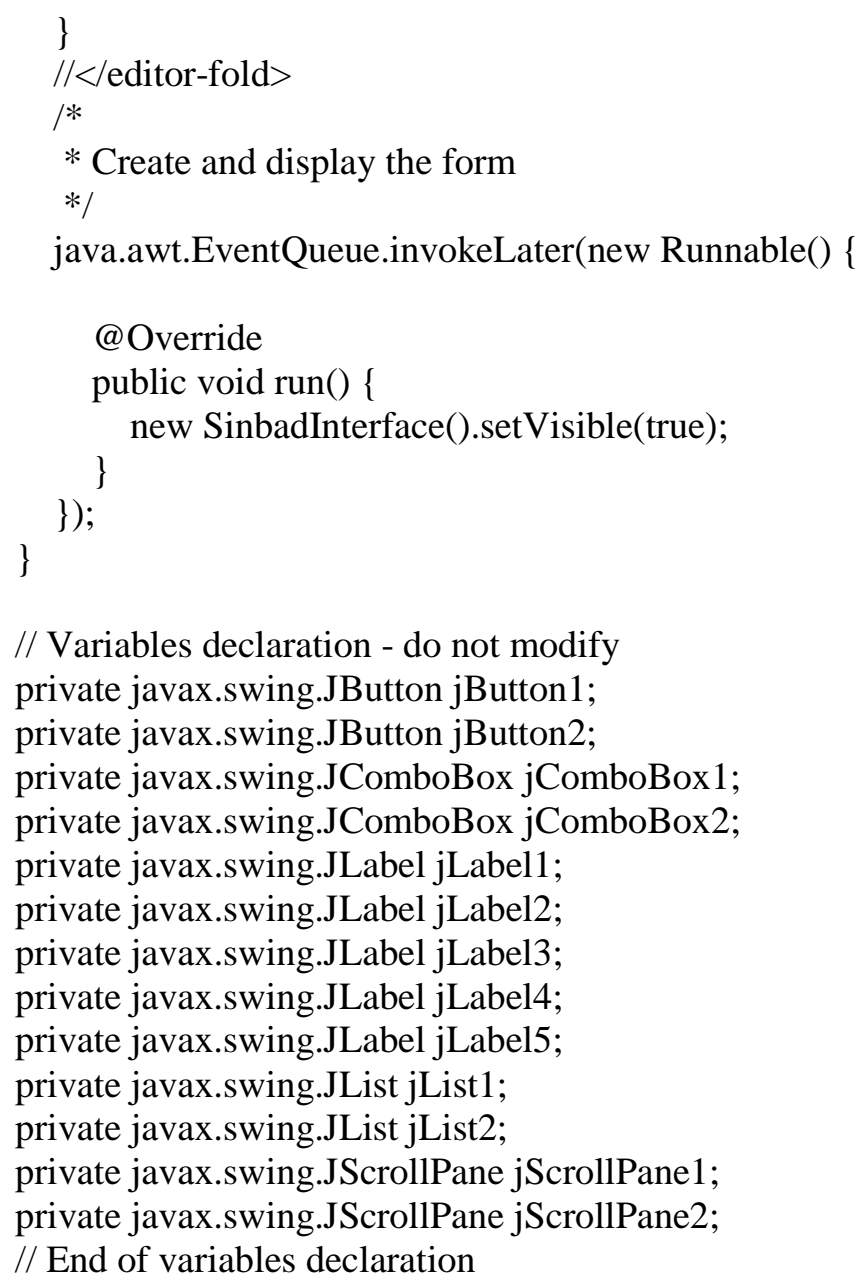

CrawlerFission.java (The search for the Reactor Shielding benchmarks type)

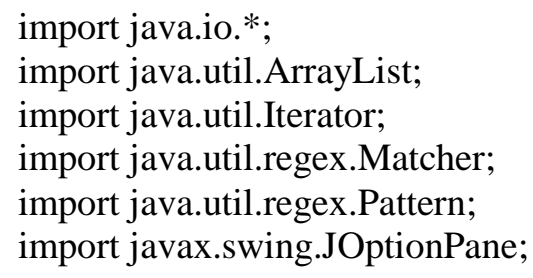

public class CrawlerFission \{

void Crawler (ArrayList key) throws FileNotFoundException, IOException \{

File arquivo;

String sourceLine;

String content = "';

String ender = "'";

ArrayList keylist $=$ key; 
String str;

ArrayList found= new ArrayList();

boolean bool=true;

try \{

File dir = null;

String home = System.getProperty("user.home");

File $\mathrm{ff}=$ new File(home);

String dire = ff.getAbsolutePath()+File.separator+".Sinbad";

if (!(new File(dire+File.separator+"fission.txt")).exists()) \{

$\operatorname{dir}=$ new File(dire);

dir.mkdir();

arquivo = new File(dir,"fission.txt");

FileOutputStream fos = new FileOutputStream(arquivo);

String ad = new File("'").getAbsolutePath();

ad=ad.replace("\\", "/");

String gg = ad+File.separator+"Docs"+File.separator+"indexfission.htm";

InputStreamReader pageInput = new InputStreamReader(new FileInputStream(gg));

BufferedReader source $=$ new BufferedReader(pageInput);

// Append each new HTML line into one string. Add a tab character.

while ((sourceLine $=$ source.readLine()) $!=$ null)

content $+=$ sourceLine + "|t";

// Remove style tags \& inclusive content

Pattern path = Pattern.compile("<a href=.*?>.*?</a >");

Matcher mpath = path.matcher(content);

while (mpath.find ()) ender += mpath + "\n";

Pattern path2 = Pattern.compile("<a href=");

Matcher mpath2 = path2.matcher(ender);

while (mpath2.find()) ender = mpath2.replaceAll("'");

Pattern path3 = Pattern.compile("</a>");

Matcher mpath3 = path3.matcher(ender);

while (mpath3.find()) ender = mpath3.replaceAll("');

Pattern path4 = Pattern.compile("<span.*?>");

Matcher mpath4 = path4.matcher(ender);

while (mpath4.find()) ender = mpath4.replaceAll("');

Pattern path7 = Pattern.compile("</span>");

Matcher mpath7 = path7. matcher(ender);

while (mpath7.find()) ender = mpath7.replaceAll("'); 


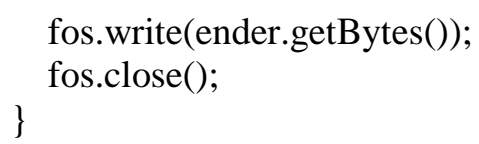

BufferedReader in = new BufferedReader(new

FileReader(home+File.separator+".Sinbad"+File.separator+"fission.txt"));

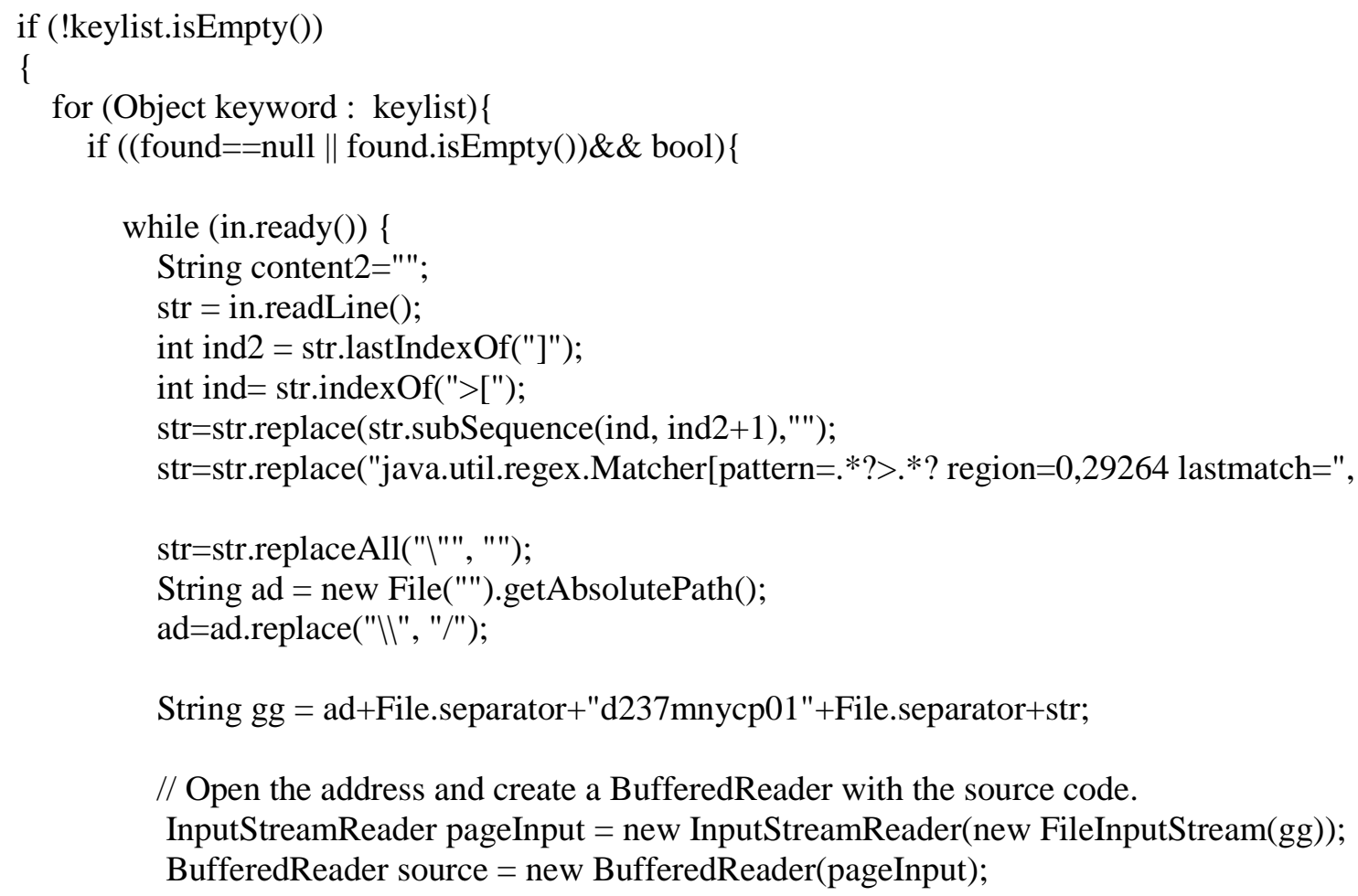




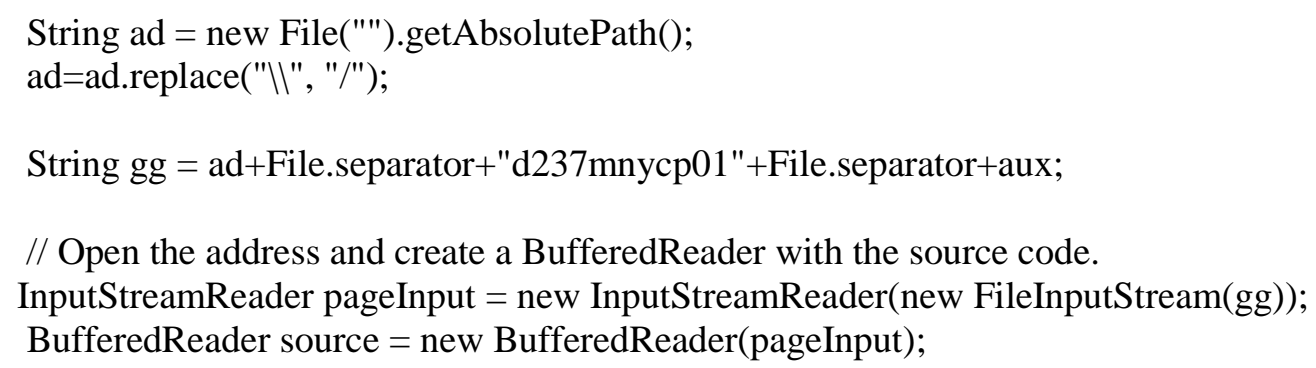


CrawlerFusion.java (The search for the Fusion Neutronics Shielding benchmarks type)

import java.io.*;

import java.util.ArrayList;

import java.util.Iterator;

import java.util.regex.Matcher;

import java.util.regex.Pattern;

import javax.swing.JOptionPane;

public class CrawlerFusion \{

void Crawler (ArrayList key) throws FileNotFoundException, IOException \{

File arquivo;

String sourceLine;

String content = "';

String ender = "';

ArrayList keylist = key;

String str;

ArrayList found= new ArrayList();

boolean bool=true;

try \{

File dir = null;

String home = System.getProperty("user.home");

File $\mathrm{ff}=$ new File(home);

String dire = ff.getAbsolutePath()+File.separator+".Sinbad";

if (!(new File(dire+File.separator+"fusion.txt")).exists()) \{

dir = new File(dire);

dir.mkdir();

arquivo = new File(dir,"fusion.txt");

FileOutputStream fos = new FileOutputStream(arquivo);

String ad = new File("').getAbsolutePath();

ad=ad.replace("|l", "/");

//URL address = new URL("file:///"+ad+"/Docs/index.htm");

String gg = ad+File.separator+"Docs"+File.separator+"indexfusion.htm";

// Open the address and create a BufferedReader with the source code.

//InputStreamReader pageInput = new InputStreamReader(address.openStream());

InputStreamReader pageInput = new InputStreamReader(new FileInputStream(gg));

BufferedReader source $=$ new BufferedReader(pageInput);

// Append each new HTML line into one string. Add a tab character.

while ((sourceLine = source.readLine()) != null)

content $+=$ sourceLine + "|t";

// Remove style tags \& inclusive content 


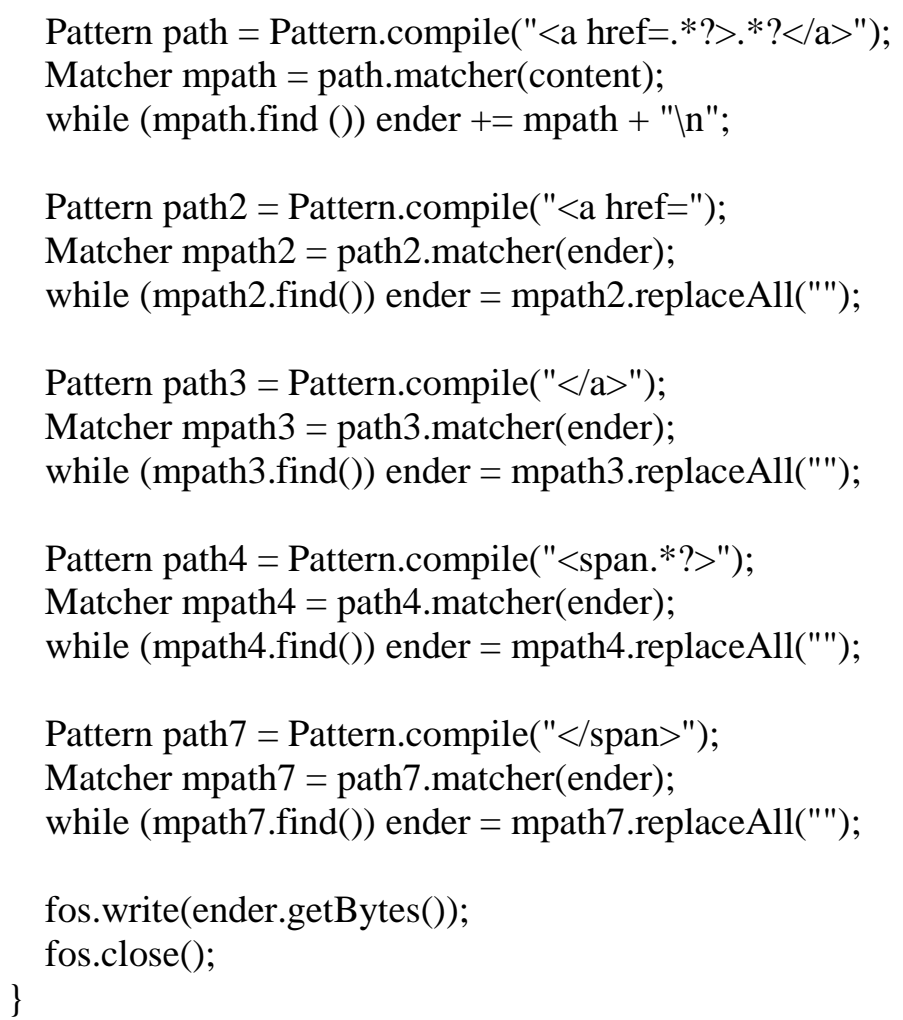

BufferedReader in = new BufferedReader(new

FileReader(home+File.separator+".Sinbad"+File.separator+"fusion.txt"));

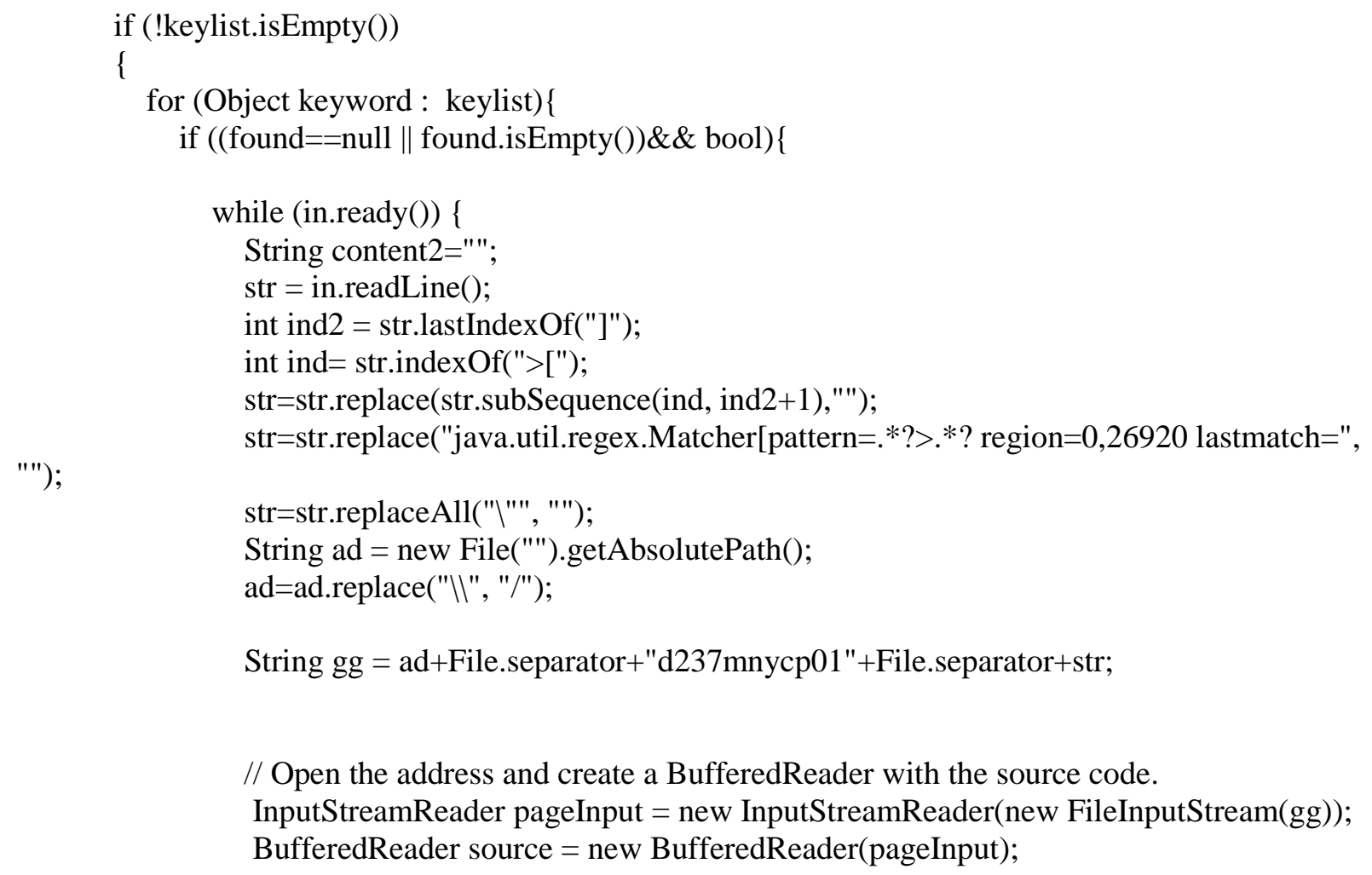

// Open the address and create a BufferedReader with the source code. InputStreamReader pageInput = new InputStreamReader(new FileInputStream(gg)); BufferedReader source $=$ new BufferedReader(pageInput); 
// Append each new HTML line into one string. Add a tab character.

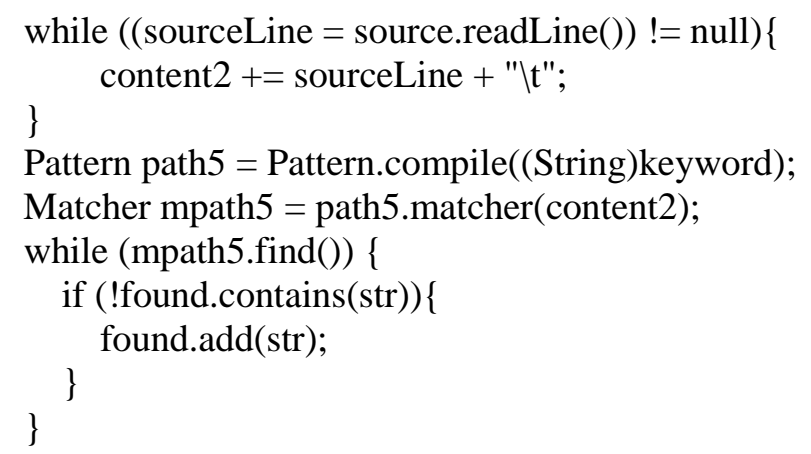

\}else $\{/ /$ if ArrayList found is not empty

bool=false;// if the list became empty after the search the cod will not go back for (Iterator $<$ String $>$ it $=$ found.iterator(); it.hasNext(); $\{$ \{

String content2="';

String aux = it.next();

String ad = new File("'").getAbsolutePath();

ad=ad.replace("\\", "/");

String gg = ad+File.separator+"d237mnycp01"+File.separator+aux;

// Open the address and create a BufferedReader with the source code.

InputStreamReader pageInput = new InputStreamReader(new FileInputStream(gg));

BufferedReader source = new BufferedReader(pageInput);

// Append each new HTML line into one string. Add a tab character.

while ((sourceLine = source.readLine()) != null) content $2+=$ sourceLine + "\t";

Pattern path6 = Pattern.compile((String)keyword);

Matcher mpath6 = path6. matcher(content2);

if (!mpath6.find()) it.remove();

\}//end intern for

\}//end else

\}//end for

\}else\{

while (in.ready()) \{

str = in.readLine();

int ind2 = str.lastIndexOf("]");

int ind = str.indexOf("> $>$ ");

str=str.replace(str.subSequence(ind, ind2+1),"');

str=str.replace("java.util.regex.Matcher[pattern=.*?>.*? region=0,26920 lastmatch=", "'); 


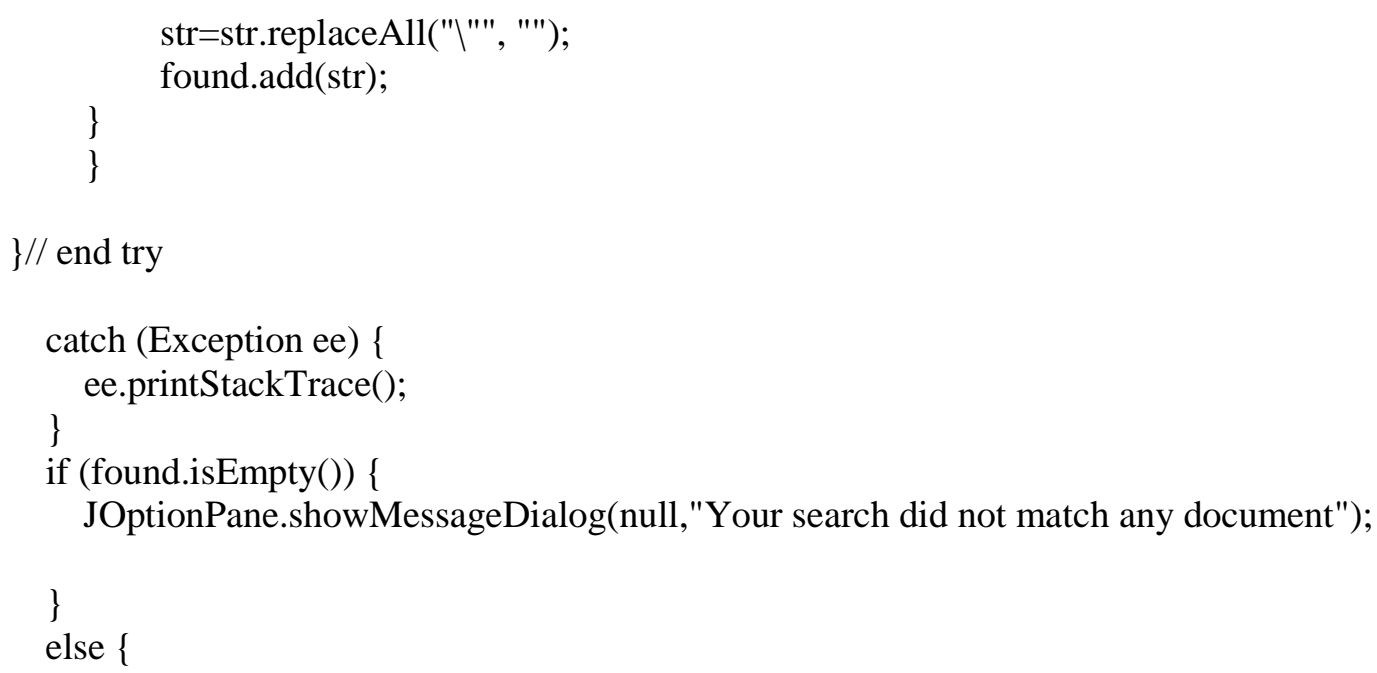

CrawlerAccelerator.java (The search for the Accelerator Shielding benchmarks type) import java.io.*; import java.util.ArrayList; import java.util.Iterator; import java.util.regex.Matcher; import java.util.regex.Pattern; import javax.swing.JOptionPane;

public class CrawlerAccelerator \{

void Crawler (ArrayList key) throws FileNotFoundException, IOException \{

File arquivo;

String sourceLine;

String content = "';

String ender = "'";

ArrayList keylist = key;

String str;

ArrayList found= new ArrayList();

boolean bool=true;

try \{

File dir = null;

String home = System.getProperty("user.home");

File ff $=$ new File(home); 
String dire = ff.getAbsolutePath()+File.separator+".Sinbad";

if (!(new File(dire+File.separator+"accelerator.txt")).exists()) \{

$\operatorname{dir}=$ new File(dire);

dir.mkdir();

arquivo = new File(dir,"accelerator.txt");

FileOutputStream fos = new FileOutputStream(arquivo);

String ad = new File("').getAbsolutePath();

ad=ad.replace("\\", "/");

String gg = ad+File.separator+"Docs"+File.separator+"indexaccelerator.htm";

// Open the address and create a BufferedReader with the source code.

//InputStreamReader pageInput = new InputStreamReader(address.openStream());

InputStreamReader pageInput = new InputStreamReader(new FileInputStream(gg));

BufferedReader source $=$ new BufferedReader(pageInput);

// Append each new HTML line into one string. Add a tab character.

while ((sourceLine $=$ source.readLine ()$) !=$ null $)$

content $+=$ sourceLine + "\t";

// Remove style tags \& inclusive content

Pattern path = Pattern.compile("<a href=.*?>.*?</a>");

Matcher mpath = path.matcher(content);

while (mpath.find ()) ender += mpath + "\n";

Pattern path2 = Pattern.compile("<a href=");

Matcher mpath2 = path2.matcher(ender);

while (mpath2.find()) ender = mpath2.replaceAll("');

Pattern path3 = Pattern.compile("</a >");

Matcher mpath3 = path3.matcher(ender);

while (mpath3.find()) ender = mpath3.replaceAll("');

Pattern path4 = Pattern.compile("<span.*?>");

Matcher mpath4 = path4.matcher(ender);

while (mpath4.find()) ender = mpath4.replaceAll("'");

Pattern path7 = Pattern.compile("</span>");

Matcher mpath7 = path7.matcher(ender);

while (mpath7.find()) ender = mpath7.replaceAll("');

fos.write(ender.getBytes());

\}

fos.close();

BufferedReader in = new BufferedReader(new

FileReader(home+File.separator+".Sinbad"+File.separator+"accelerator.txt")); 


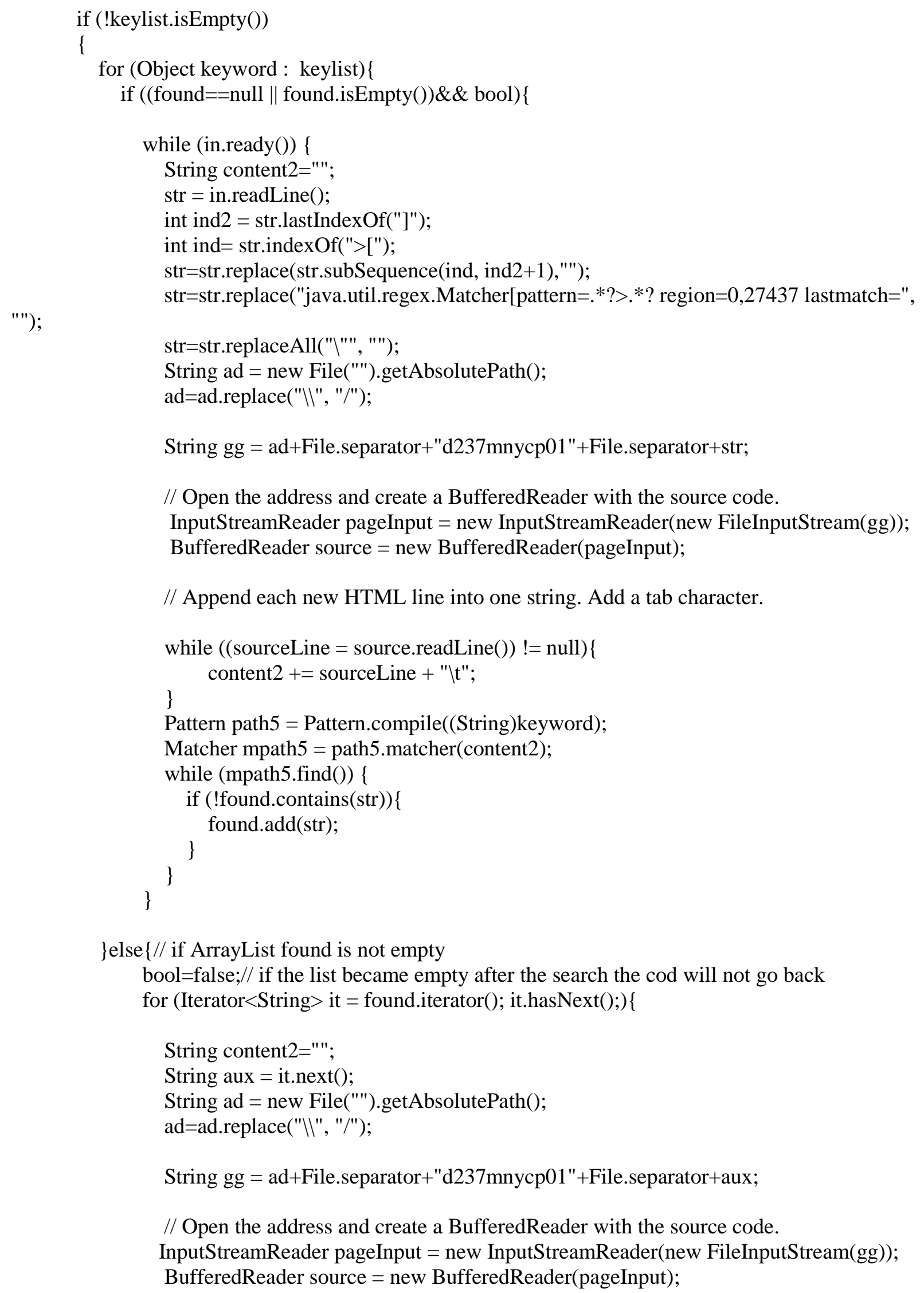

\}else $\{/ /$ if ArrayList found is not empty

bool=false;// if the list became empty after the search the cod will not go back for (Iterator $<$ String $>$ it $=$ found.iterator(); it.hasNext(); \{

String content2="'";

String aux = it.next();

String ad = new File("'").getAbsolutePath();

ad=ad.replace("\\", "/");

String gg = ad+File.separator+"d237mnycp01"+File.separator+aux;

// Open the address and create a BufferedReader with the source code.

InputStreamReader pageInput = new InputStreamReader(new FileInputStream(gg)); BufferedReader source $=$ new BufferedReader(pageInput); 
// Append each new HTML line into one string. Add a tab character.

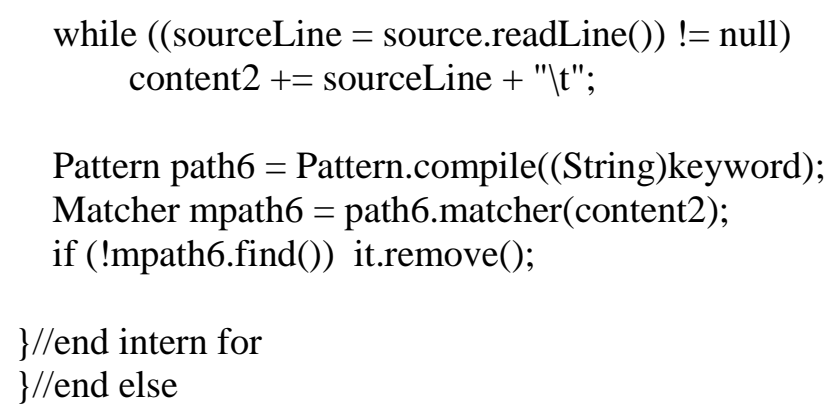

CrawlerAll.java (The search All types of benchmarks)

import java.io.*;

import java.util.ArrayList;

import java.util.Iterator;

import java.util.regex.Matcher;

import java.util.regex.Pattern; 
import javax.swing.JOptionPane;

public class CrawlerAll \{

void Crawler (ArrayList key) throws FileNotFoundException, IOException \{

File arquivo;

String sourceLine;

String content = "';

String ender = "'";

ArrayList keylist = key;

String str;

ArrayList found= new ArrayList();

boolean bool=true;

try \{

File dir = null;

String home = System.getProperty("user.home");

File ff = new File(home);

String dire = ff.getAbsolutePath()+File.separator+".Sinbad";

if (!(new File(dire+File.separator+"file.txt")).exists()) \{

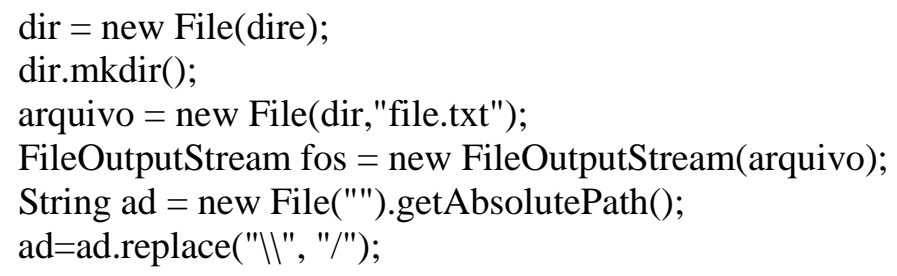

String gg = ad+File.separator+"Docs"+File.separator+"index.htm";

InputStreamReader pageInput = new InputStreamReader(new FileInputStream(gg)); BufferedReader source $=$ new BufferedReader(pageInput);

// Append each new HTML line into one string. Add a tab character.

while ((sourceLine $=$ source.readLine()) $!=$ null) content += sourceLine + "|t";

// Remove style tags \& inclusive content

Pattern path = Pattern.compile("<a href=.*?>.*?</a>");

Matcher mpath = path.matcher (content);

while (mpath.find ()) ender += mpath + "\n";

Pattern path2 = Pattern.compile("<a href=");

Matcher mpath2 = path2. matcher(ender);

while (mpath2.find()) ender = mpath2.replaceAll("'); 


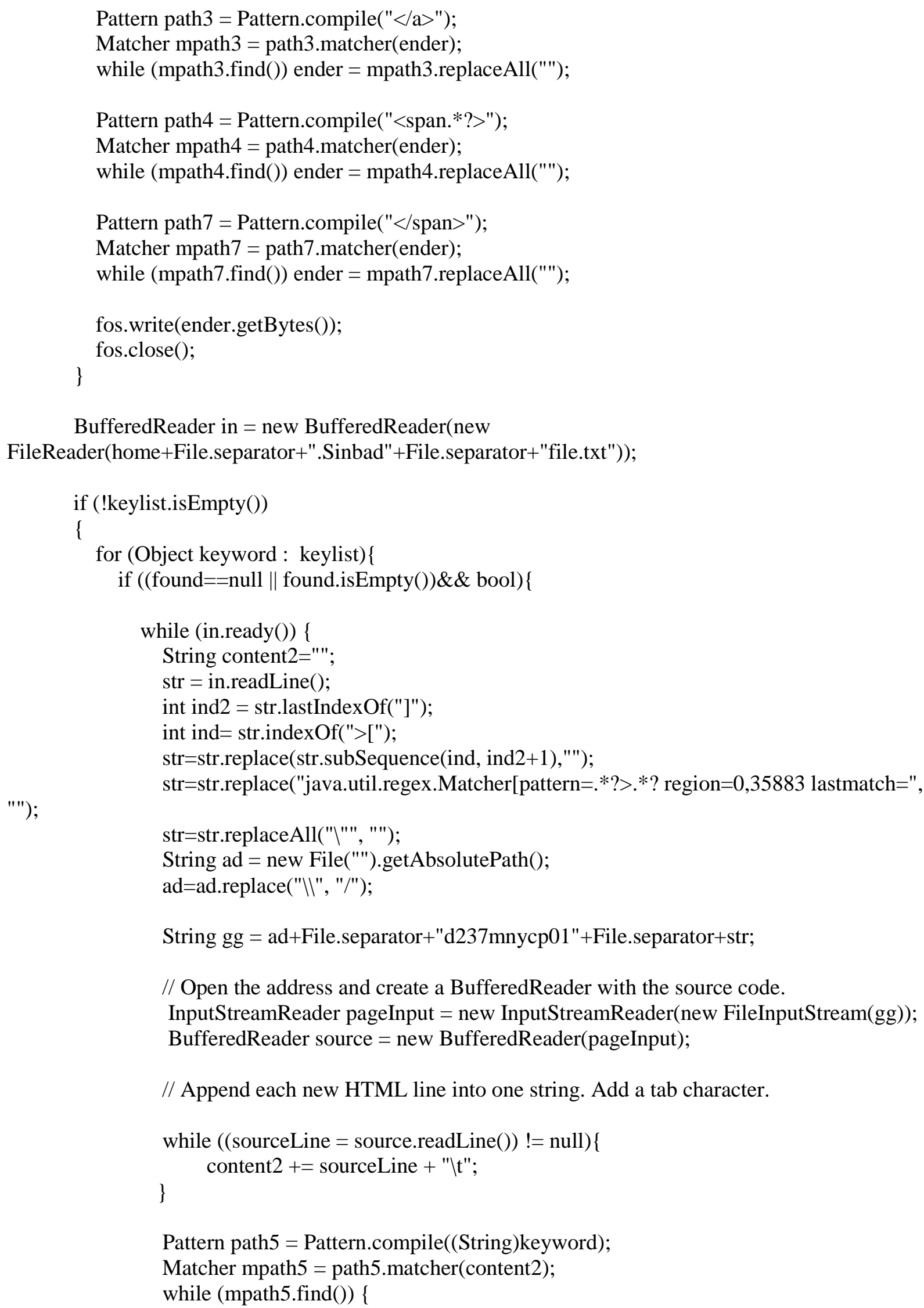

BufferedReader in = new BufferedReader(new

FileReader(home+File.separator+".Sinbad"+File.separator+"file.txt"));

if (!keylist.isEmpty())

\{

for (Object keyword : keylist) \{

if ((found==null || found.isEmpty())\&\& bool) \{

while (in.ready()) \{

String content $2=" '$;

str $=$ in.readLine ()$;$

int ind2 = str.lastIndexOf("]");

int ind= str.indexOf(">[");

str=str.replace(str.subSequence(ind, ind2+1),"');

str=str.replace("java.util.regex.Matcher[pattern=.*?>.*? region=0,35883 lastmatch=",

str=str.replaceAll("'"'", "');

String ad = new File("'").getAbsolutePath();

ad=ad.replace("lı", "/");

String gg = ad+File.separator+"d237mnycp01"+File.separator+str;

// Open the address and create a BufferedReader with the source code.

InputStreamReader pageInput = new InputStreamReader(new FileInputStream(gg));

BufferedReader source = new BufferedReader(pageInput);

// Append each new HTML line into one string. Add a tab character.

Pattern path5 = Pattern.compile((String)keyword);

Matcher mpath5 = path5.matcher(content2);

while (mpath5.find()) \{

"'); 


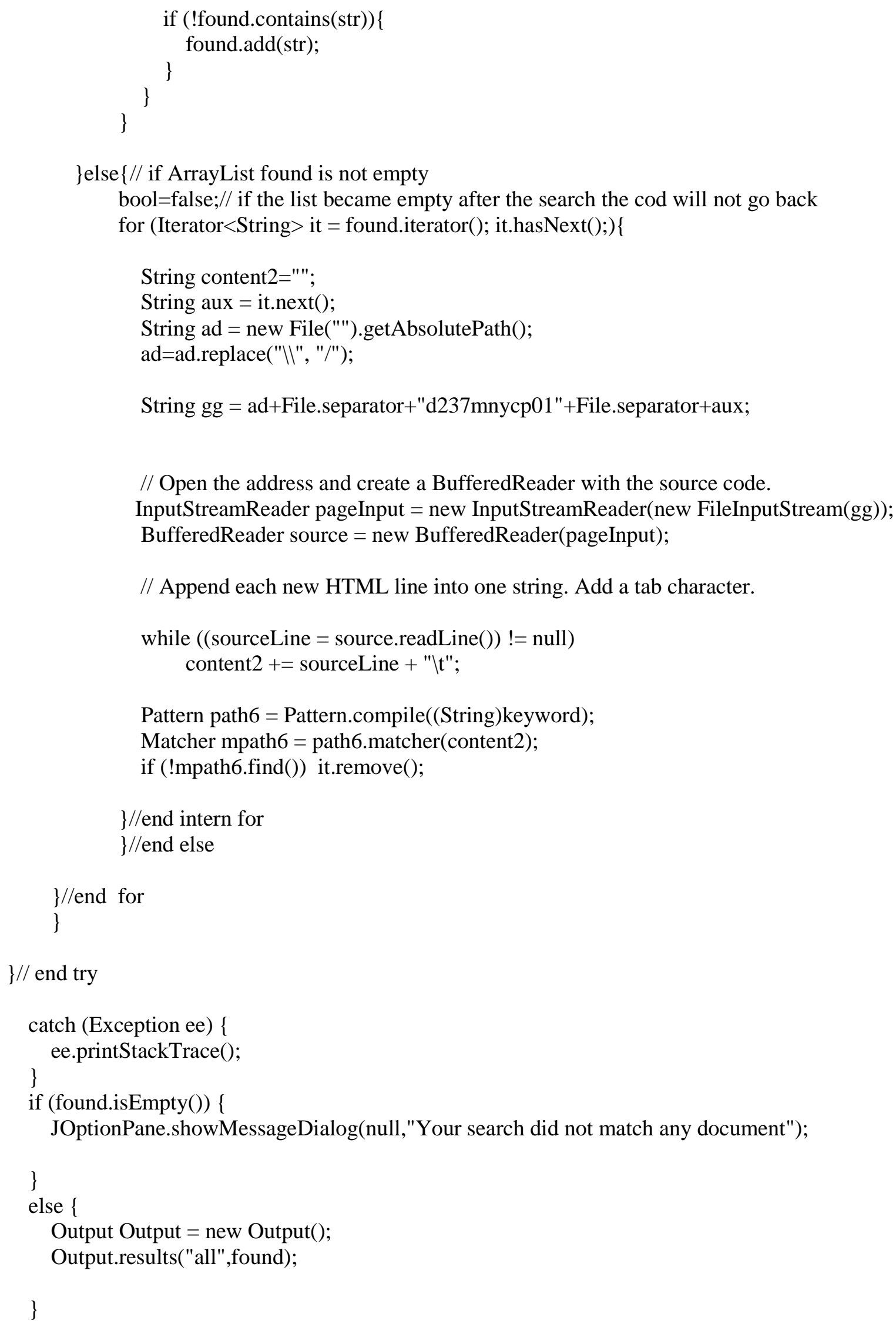




\section{Output.java (Organize the results)}

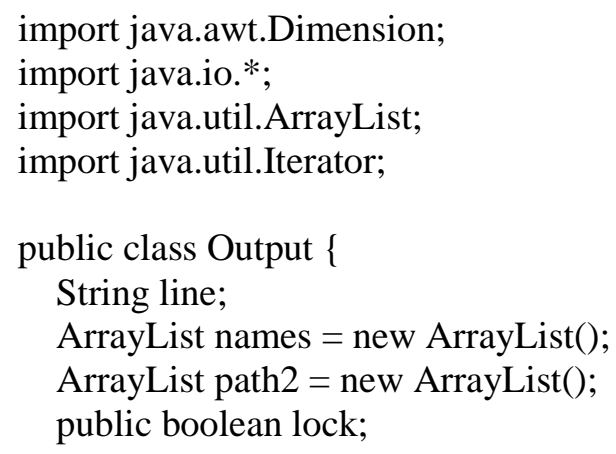

void results (String nam,ArrayList path) throws FileNotFoundException, IOException \{ String home = System.getProperty("user.home");

String name $=$ nam;

path2=path;

for (Iterator $<$ String $>$ it $=$ path2. iterator(); it.hasNext(); \{

String aux = it.next();

if ("all".equals(name) $)\{$

BufferedReader in = new BufferedReader(new

FileReader(home+File.separator+".Sinbad"+File.separator+"file.txt"));

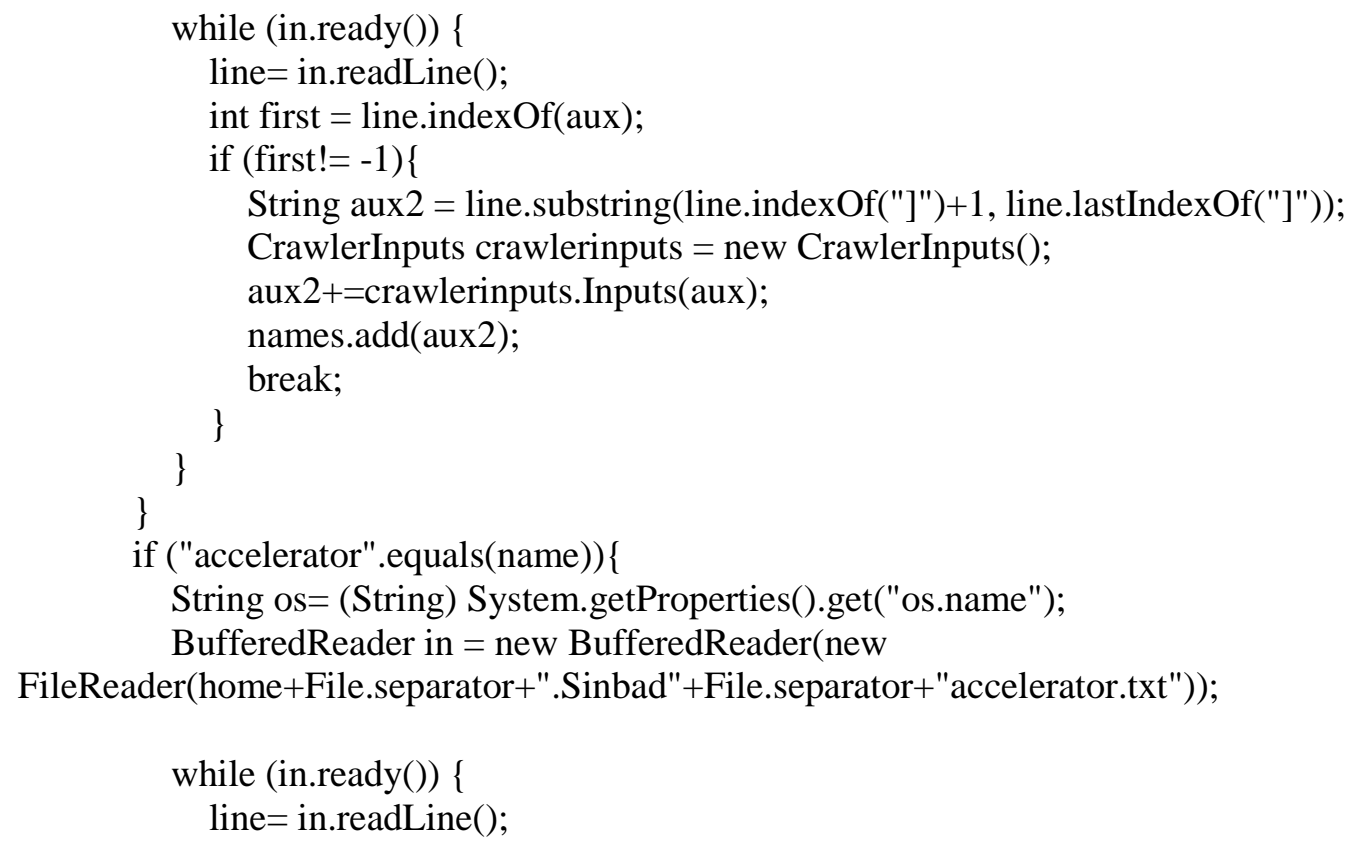




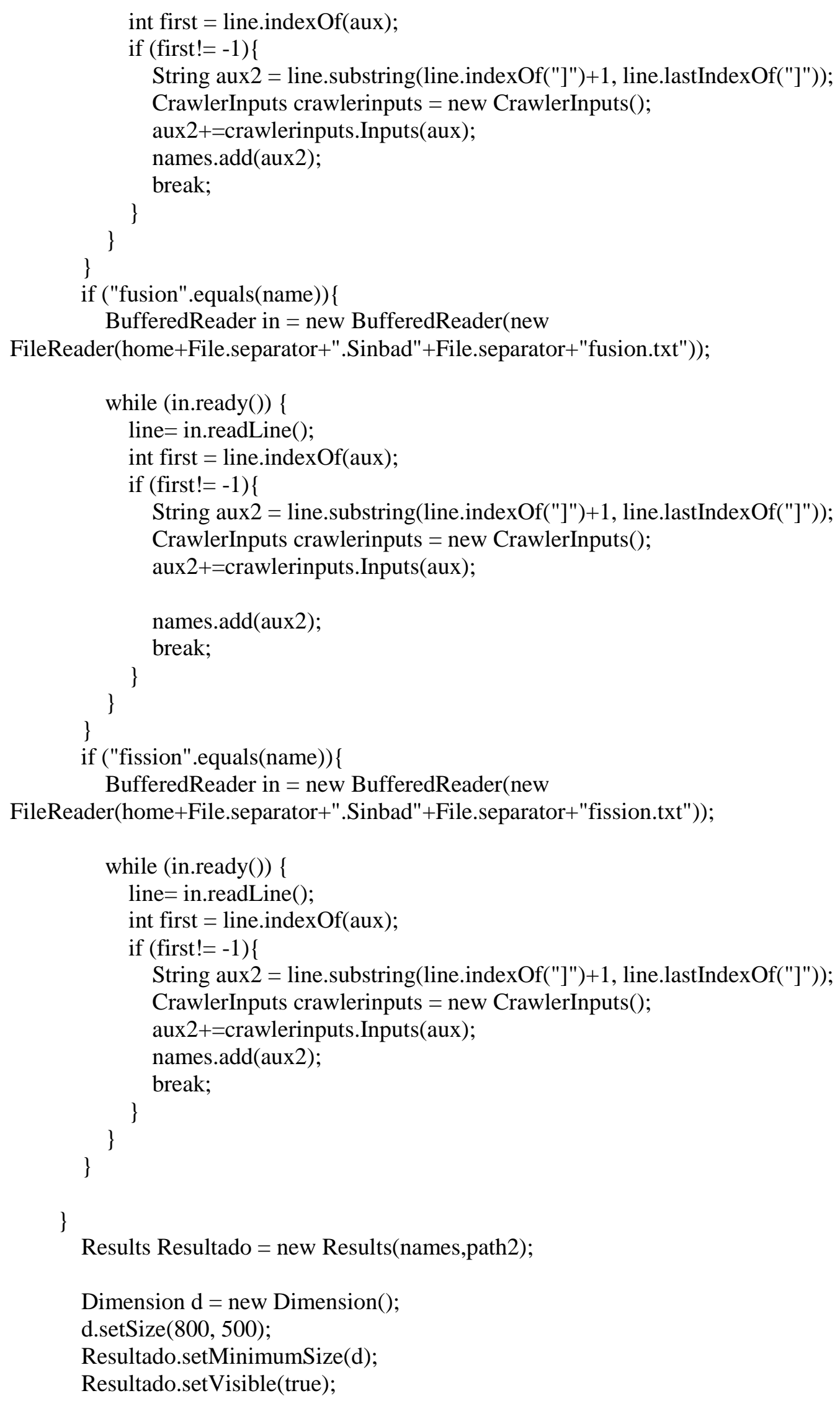


CrawlerInputs.java ( Returns the kind of input the benchmarks has)

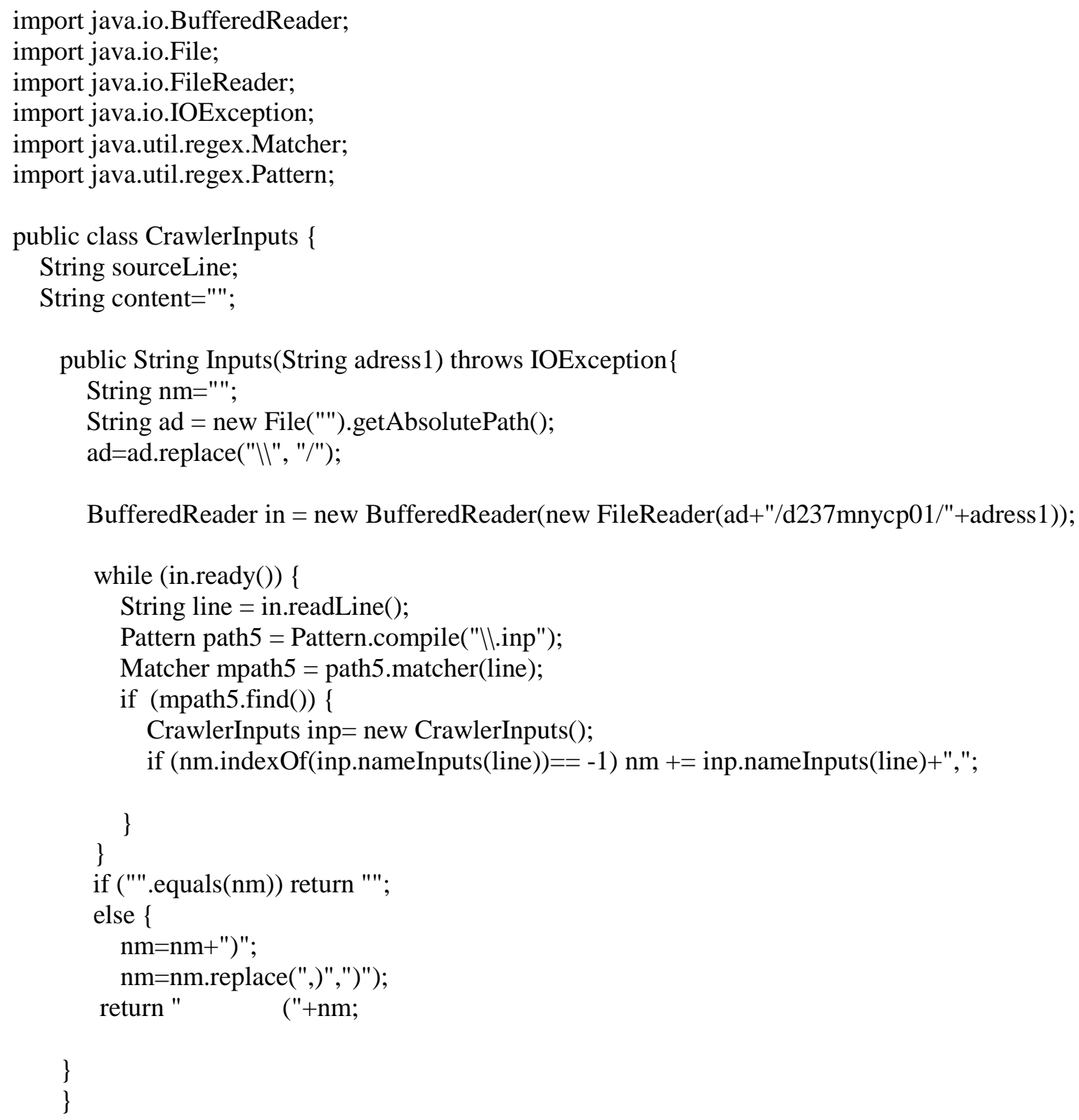

public String nameInputs(String line) throws IOException\{

String method="'";

Pattern path = Pattern.compile("Input data for");

Matcher mpath = path.matcher(line);

if (mpath.find()) \{

if (line.indexOf("Input data for")!=-1) \{

int begin=line.indexOf("Input data for")+14; 


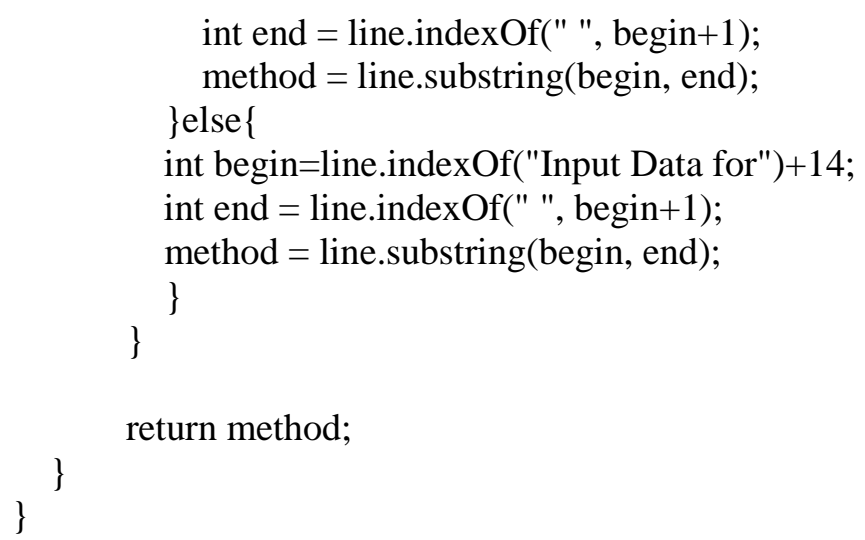

Results.java (The results interface)

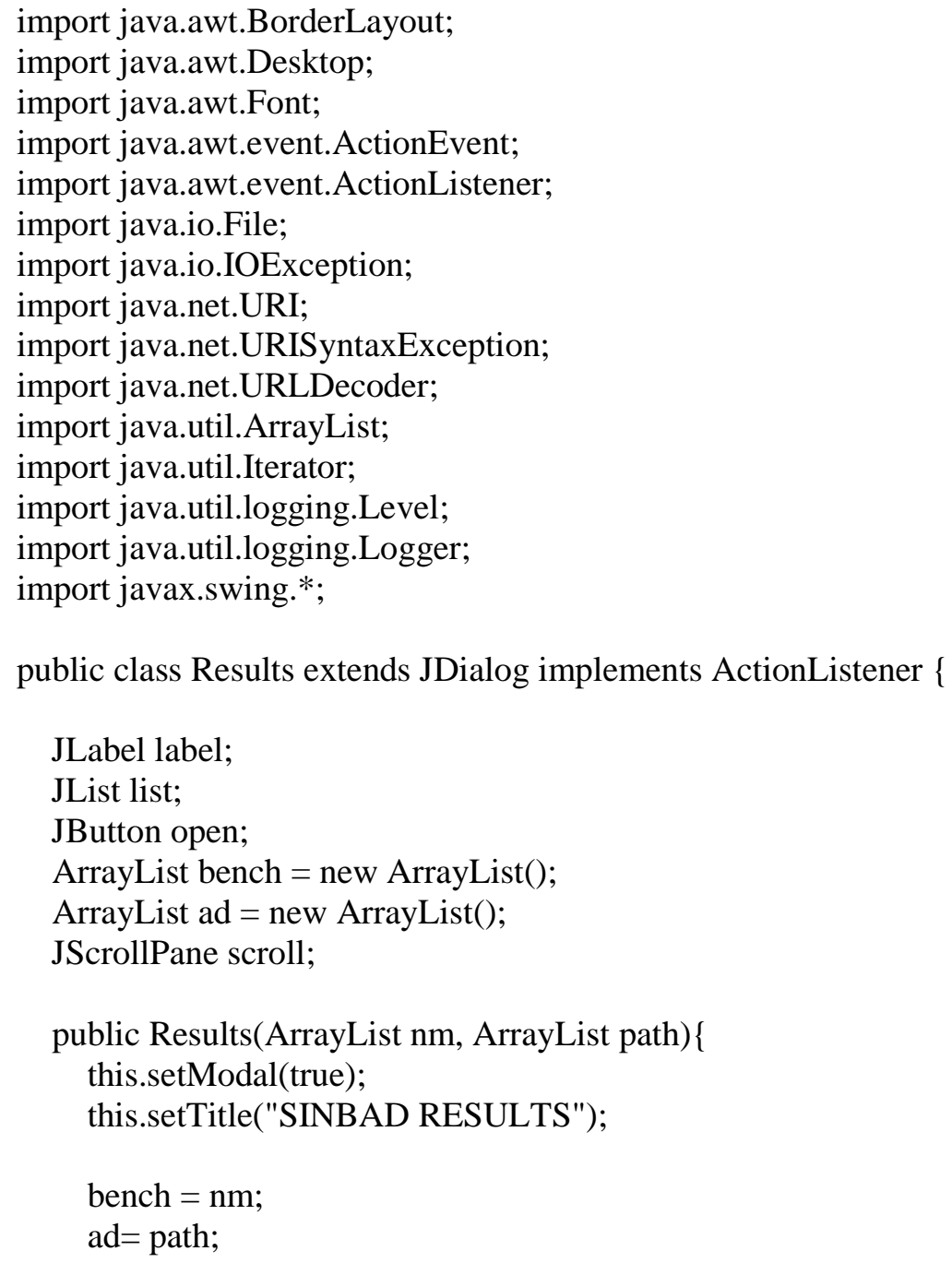




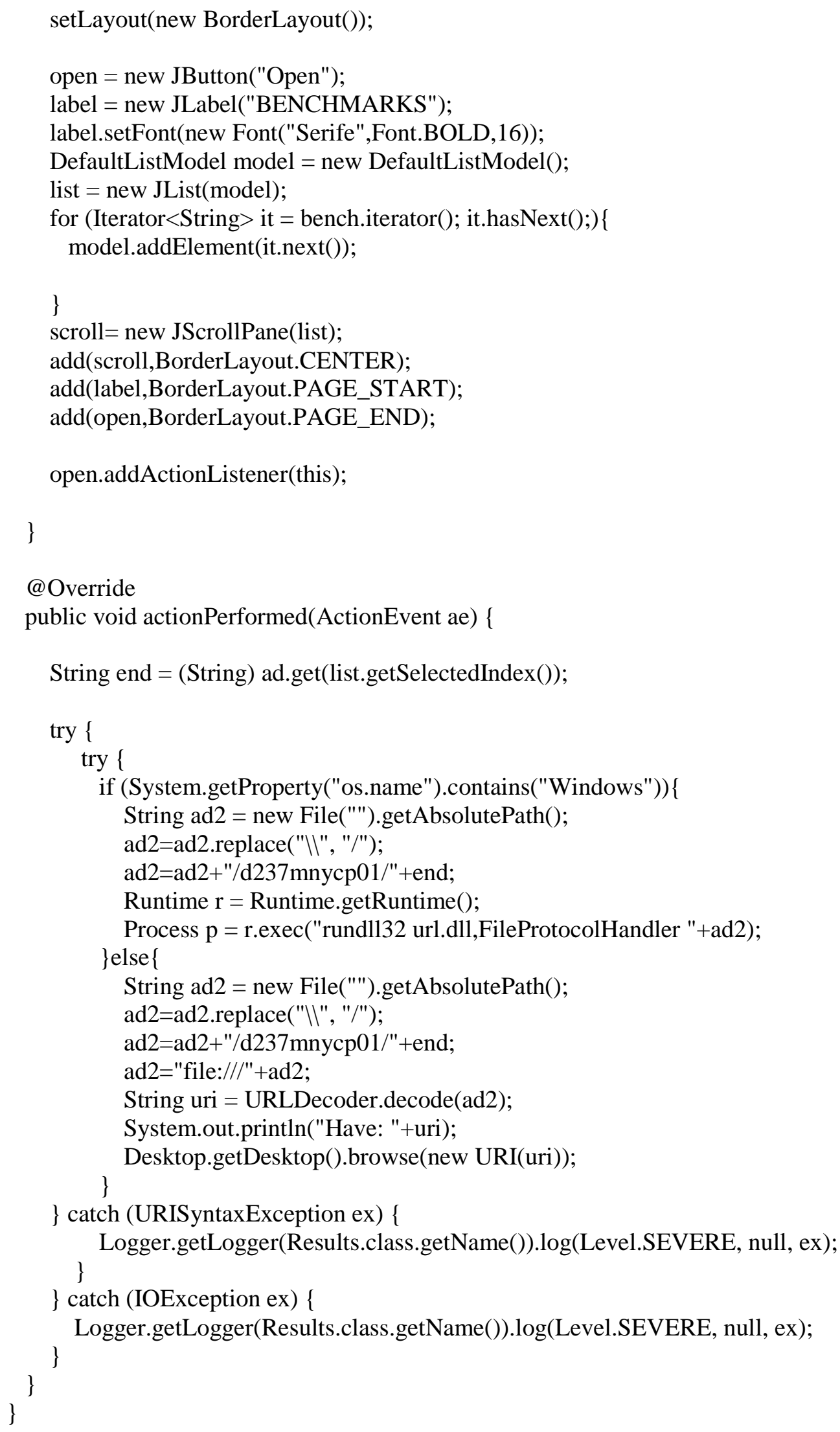


B-30 